\title{
MANAGING CONSENSUS BY MULTI-STAGE OPTIMIZATION MODELS WITH LINGUISTIC PREFERENCE ORDERINGS AND DOUBLE HIERARCHY LINGUISTIC PREFERENCES
}

\author{
Xunjie GOU ${ }^{1}$, Zeshui XU1 ${ }^{*}$, Wei ZHOU(1) 2\# \\ ${ }^{1}$ Business School, Sichuan University, Chengdu 610064, China \\ ${ }^{2}$ School of Finance, Yunnan University of Finance and Economics, Kunming 650221, China
}

Received 16 August 2019; accepted 23 November 2019

\begin{abstract}
Preference ordering structures are useful and popular tools to represent experts' preferences in the decision making process. In the existing preference orderings, they lack the research on the precise relationship between any two adjacent alternatives in the preference orderings, and the decision making methods are unreasonable. To overcome these issues, this paper establishes a novel concept of linguistic preference ordering (LPO) in which the ordering of alternatives and the relationships between two adjacent alternatives should be fused well, and develops two transformation models to transform each LPO into the corresponding double hierarchy linguistic preference relation with complete consistency. Additionally, to fully respect the experts' expression habits and provide more refined solutions to experts, this paper establishes a multi-stage consensus optimization model by considering the suggested preferences represented in both the continuous scale and the discrete scale, and develops a multi-stage interactive consensus reaching algorithm to deal with multi-expert decision making problem with LPOs. Furthermore, some numerical examples are presented to illustrate the developed methods and models. Finally, some comparative analyses between the proposed methods and models and some existing methods have been made to show the advantages of the proposed methods and models.
\end{abstract}

Keywords: linguistic preference orderings, double hierarchy linguistic preference relations, consensus, multi-stage optimization models, multi-expert decision making.

JEL Classification: C44, C61, C63, D70, D81.

\section{Introduction}

Multi-expert decision making (MEDM) (or Group decision making) can be regarded as a situation in which a group of experts are invited to provide their individual opinions by evaluating the given alternatives, and then select the optimal alternative(s). As useful and popular tools, preference ordering structures have been proposed by some scholars (Chiclana et al., 1998; González-Pachón \& Romero, 2001; He \& Xu, 2018; Hervés-Beloso \& Cruces,

*Corresponding author. E-mail: xuzeshui@263.net

\#Corresponding author. E-mail: zw453@163.com

(c) 2020 The Author(s). Published by VGTU Press

This is an Open Access article distributed under the terms of the Creative Commons Attribution License (http://creativecommons. org/licenses/by/4.0/), which permits unrestricted use, distribution, and reproduction in any medium, provided the original author and source are credited. 
2018; Liang et al., 2018; Schubert, 1995; Tanino, 1984; Xu, 2013; Zhang et al., 2018), which have been used to express experts' preferences regarding all alternatives by ordering structures. Considering that experts may have different expression habits, knowledge backgrounds and cognitive levels, the preference ordering structures provided by them may be represented by different forms such as preference orderings (Chiclana et al., 1998; Schubert, 1995; He \& $\mathrm{Xu}, 2018$; Zhang et al., 2018), interval preference orderings (González-Pachón \& Romero, 2001), fuzzy preference orderings (Beliakov et al., 2017; Tanino, 1984), continuous preference orderings (Hervés-Beloso \& Cruces, 2018), hesitant preference ordering sets (He \& Xu, $2018)$, etc. For instance, suppose that $\left\{A_{1}, A_{2}, A_{3}, A_{4}\right\}$ is a set of alternatives, then $\{2,1,4,3\}$ is a preference ordering in which the positive integers are used by the experts to show the order positions of alternatives. Similarly, other preference orderings can be represented by different forms of preference information such as interval positive integers, hesitant fuzzy numbers, etc.

However, two critical problems about the existing preference orderings have not yet been fully addressed:

(1) The existing preference orderings can only reflect the ranking ordering of alternatives, but lack the research on the precise relationship between any two adjacent alternatives in the preference orderings. In other words, the existing preference orderings default to the same relationship between two adjacent alternatives in the preference orderings (Chiclana et al., 1998). For example, when evaluating the comprehensive quality of three cars $\left\{A_{1}, A_{2}, A_{3}\right\}$, the expert may say " $A_{2}$ is very better than $A_{1}$, and $A_{1}$ is slightly better than $A_{3}$ ". However, we can only obtain the preference ordering $\{2,1,3\}$, but ignore the words that involve the relationships between any two adjacent alternatives such as "very better" and "slightly better". Therefore, to represent preferences more comprehensively and correctly, the preference ordering should include not only the ordering of alternatives, but also the relationships between any two adjacent alternatives in preference ordering.

(2) The existing methods mainly aggregate the preference orderings and then obtain the final ordering of all alternatives ( $\mathrm{He} \& \mathrm{Xu}, 2018)$. However, because the relationships between any two adjacent alternatives are usually unbalanced, so there is an urgent need to deal with the preference orderings that contain both the ordering of alternatives and the relationships between two adjacent alternatives.

To overcome the first problem, it is necessary to establish a novel preference ordering structure in which the ordering of alternatives and the relationships between two adjacent alternatives should be fused well. Considering that natural languages are more in line with the real thoughts of people, we can use linguistic information to represent the relationships between two adjacent alternatives. In recent years, lots of linguistic representation models have been developed such as simple linguistic representation model (Zadeh, 1975), virtual linguistic representation model (Xu, 2005), 2-tuple linguistic model (Herrera \& Martínez, 2000), etc. However, when expressing some complex linguistic information, there are some gaps. For instance, some linguistic models only contain parts of the original linguistic information, and some linguistic labels or numerical scales have no clear meaning. To overcome these gaps, double hierarchy linguistic term set (DHLTS) was defined by Gou et al. (2017), 
and it can be used to handle complex linguistic information well by dividing them into two simple linguistic hierarchies where the first hierarchy linguistic term set (LTS) is the main linguistic hierarchy and the second hierarchy LTS is the linguistic feature or detailed supplementary of each linguistic term in the first hierarchy LTS (Gou et al., 2017). Therefore, in this paper, we develop a novel concept of linguistic preference ordering (LPO) by combining the preference ordering and double hierarchy linguistic terms (DHLTs, the basic elements of DHLTS). Additionally, depending on the interests of the experts, there exist two situations: One is that all alternatives are in a preference ordering, which is called the LPO in continuous form; The other one is that a set contains several preference orderings, and each of them only consists of the relationship of two alternatives, which is called the LPO in decentralized form. Therefore, we will focus on dealing with two kinds of LPOs in continuous form and in decentralized form.

To overcome the second problem, this paper introduces the concept of double hierarchy linguistic preference relation (DHLPR) (Gou et al., 2019). Then we can equivalently transform each LPO into the corresponding DHLPR with complete consistency. Additionally, we can obtain the final decision making result by proposing some consensus models with DHLPRs, which is equal to the decision making result with LPO information.

For achieving consensus among a group of experts, lots of consensus reaching approaches and models over preference relations have been developed in recent years (Del Moral et al., 2018; Gou et al., 2018a; Kacprzyk \& Fedrizzi, 1988; Kamis et al., 2018; Lan et al., 2018; Liao et al., 2016; Morente-Molinera et al., 2018, 2019; Wu et al., 2018; Zhang \& Chen, 2019; Zhu \& $\mathrm{Xu}, 2018)$. However, most of the consensus models are generally iterative models that utilize heuristics as their calculation tools such as some automatic improvement models (Gou et al., 2018a; Liao et al., 2016; Zhang \& Chen, 2019) and feedback mechanism-based improvement models (Gou et al., 2018a; Liao et al., 2016; Wu et al., 2018). Therefore, the most important defect of these consensus models mentioned above is that we have to go through trial and error before reaching a consensus or even failing to reach a consensus.

In contrast, some scholars developed another popular tool named as optimization model to help experts achieve consensus (Ben-Arieh \& Easton, 2007; Fan et al., 2006; Meng et al., 2019; Wan et al., 2018; Wu et al., 2019a, 2019b; Xu et al., 2018; Yu \& Xu, 2020; Zhang et al., 2018a, 2019; Zhang \& Pedrycz, 2018). As far as we know, the most important advantage of optimization models is that they can provide the adjusted preference solutions directly by setting goals and solving the established models. Therefore, these adjusted preference solutions are optimal to a certain degree of objectivity, and can be understood and accepted by experts in a feedback strategy. Zhang and Pedrycz (2018) built several goal programming models to manage consistency and consensus of intuitionistic multiplicative preference relations. Furthermore, the interactive consistency process and interactive consensus process based on the multi-stage models are also designed (Wu et al., 2019a). Fan et al. (2006) constructed a linear goal programming model to integrate the two different formats of preference relations and to achieved consensus. Zhang, Liang, Gao and Zhang (2018a) proposed a consensusoriented aggregation model to obtain a collective opinion with maximum consensus degree by minimizing the information deviation between individual and collective opinions. $\mathrm{Wu}$, Ren and Xu (2019b) constructed an optimization model to directly identify hesitant fuzzy 
linguistic preference values, which greatly improves the efficiency of the consensus reaching process. Zhang et al. (2019) developed a multi-stage optimization-based consensus reaching processes, which have better comprehensive consensus efficiency in different group decision making settings.

Although different kinds of optimization methods and models have been developed to solve consensus issues for different preference relations. However, two key problems have not been adequately addressed.

Firstly, when feedbacking modification suggestions to the corresponding experts in consensus reaching process, the suggested preferences for improving consensus are usually represented in continuous forms ( $\mathrm{Xu}$ et al., 2016). However, these preferences with continuous forms usually have no clear meanings considering that the original preference terms have been expressed by discrete terms (Kou \& Lin, 2014). Thus, it is very interesting and challenging to research the correspondence between the suggested preferences and the given original discrete terms. Additionally, some scholars also developed some models in which the suggested preferences are represented by discrete scales ( $\mathrm{Wu} \& \mathrm{Xu}, 2018)$. Therefore, motivated by $\mathrm{Wu}$, Huang and $\mathrm{Xu}$ (2019a) and to fully respect the experts' expression habits, in this paper, we focus on establishing some consensus optimization models by considering the suggested preferences represented in both the continuous scale and the discrete scale.

Secondly, most of the existing optimization models only focus on minimizing the size of change for preferences and did not consider the uniqueness of the obtained solutions. As far as we know, there usually are multiple optimal solutions when solving an optimization model, so it is unreasonable to consider only one aspect of them. Therefore, to provide more refined solutions to experts, this paper develops a multi-stage consensus optimization model which consists of three objectives including minimizing the deviations of the modification magnitudes, minimizing the cardinal number of modifications while keeping the value of the first objective constant, and minimizing the number of experts who need to change their evaluations.

The main innovation points of this paper are highlighted as follows:

(1) By combining the preference ordering and double hierarchy linguistic terms (DHLTs), we develop two novel concepts of LPOs, which are in continuous form and in decentralized form, respectively.

(2) Two equivalent transformation models are developed to transform LPOs into the corresponding DHLPRs with complete consistencies.

(3) A multi-stage consensus optimization model is established by considering suggested preferences represented in both the continuous scale and the discrete scale. Additionally, the proposed optimization model can optimize three objectives simultaneously.

(4) Some comparative analyses are discussed to show the advantages of the equivalent transformation models used to transform the LPOs into the corresponding DHLPRs with complete consistencies and the proposed optimization model.

The remainder of the paper is organized as follows: Section 1 reviews some related concepts of preference ordering and DHLPR. Section 2 discusses two kinds of LPOs, and develops two transformation models to transform LPOs into DHLPRs. Section 3 establishes 


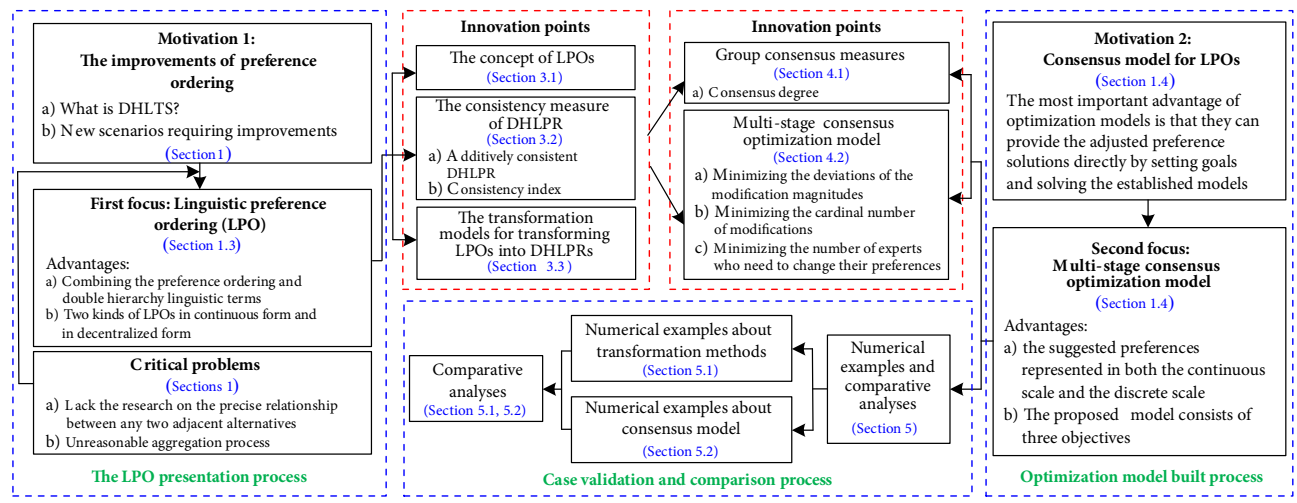

Figure 1. The main work of this paper

a multi-stage consensus optimization model and sets up an interactive consensus reaching algorithm with LPOs. Section 4 gives some numerical examples and comparative analyses with the existing methods. Some concluding remarks are summarized in last section. A flow chart can be drawn to show the main work of this paper in Figure 1.

\section{Preliminaries}

As the basis of this paper, some related concepts are reviewed in this section including preference ordering and DHLPR.

\subsection{Preference ordering}

In the decision making process, many experts may like to provide their preferences using preference orderings of alternatives. Recently, lots of preference orderings have been proposed (Chiclana et al., 1998; González-Pachón \& Romero, 2001; He \& Xu, 2018; HervésBeloso \& Cruces, 2018; Liang et al., 2018; Schubert, 1995; Tanino, 1984; Xu, 2013; Zhang et al., 2018b). Let $A=\left\{A_{1}, A_{2}, \ldots, A_{m}\right\}$ be a set of alternatives, $E=\left\{e^{1}, e^{2}, \ldots, e^{n}\right\}$ be a set of experts. Then the concept of classical preference ordering (Chiclana et al., 1998) are shown as follows: An expert $e^{k}$ provides his/her preference on $A$ as a preference ordering, $O^{k}=\left\{o_{1}^{k}, o_{2}^{k}, \ldots, o_{m}^{k}\right\}(k=1,2, \ldots, n)$, where $o_{i}^{k}(i=1,2, \ldots, m)$ denote the positional orders of the alternatives $A_{i}(i=1,2, \ldots, m)$.

To deal with preference orderings in actual decision making, some scholars developed several transformation functions to transform preference ordering into fuzzy preference relation (FPR). In general, a FPR $P=\left(p_{i j}\right)_{m \times m}$, should satisfy $p_{i j} \in[0,1]$ and $p_{i j}+p_{j i}=1$.

Chiclana et al. (1998) developed two functions, denoted as $g^{1}$ and $g^{2}$, to transform preference ordering $O^{k}$ into the FPR $P^{k}=\left(p_{i j}^{k}\right)_{m \times m}$ :

(1) $g^{1}: p_{i j}^{k}=g^{1}\left(o_{j}^{k}-o_{i}^{k}\right)=\left\{\begin{array}{ll}1 & \text { if } o_{j}^{k}>o_{i}^{k} \\ 0 & \text { if } o_{i}^{k}<o_{j}^{k}\end{array} \quad(i \neq j)\right.$; 
(2) $g^{2}: p_{i j}^{k}=g^{2}\left(o_{j}^{k}-o_{i}^{k}\right)=\left\{\begin{array}{ll}1 & \text { if } o_{j}^{k}>o_{i}^{k} \\ \frac{1}{2} & \text { if } o_{j}^{k}=o_{i}^{k} \\ 0 & \text { if } o_{j}^{k}<o_{i}^{k}\end{array}\right.$;

(3) By defining the concept of utility value $u_{i}=\frac{m-o_{i}^{k}}{m-1}$, Dombi (1995) utilized a function $g^{3}$ to transform preference ordering $O^{k}$ into the FPR $P^{k}$, where $p_{i j}^{k}=g^{3}\left(u_{i}-u_{j}\right)=\frac{1}{2}\left(1+u_{i}-u_{j}\right) ;$

(4) By the utility values given by Tanino (1988), Chiclana et al. (1998) utilized a function $g^{4}$ to transform the preference ordering $O^{k}$ into the FPR $P^{k}$, where $p_{i j}^{k}=g^{4}\left(o_{j}^{k}-o_{i}^{k}\right)=\frac{1}{2}\left(1+\frac{o_{j}^{k}}{m-1}-\frac{o_{i}^{k}}{m-1}\right)$.

\subsection{Double hierarchy linguistic preference relation}

Considering that people usually use natural languages to talk with others, express emotions or comments on something, etc. Therefore, natural languages are in line with the real thoughts of experts and Zadeh (1975) proposed fuzzy linguistic approach to deal with them. As we mentioned in Section 1, DHLTS (Gou et al., 2017), as a popular complex linguistic representation tool, can be used to represent complex linguistic information directly based on two hierarchy LTSs. Let $S=\left\{s_{t} \mid t=-\tau, \ldots,-1,0,1, \ldots, \tau\right\}$ be the first hierarchy LTS, and $O^{t}=\left\{o_{k}^{t} \mid k=-\varsigma, \ldots,-1,0,1, \ldots, \varsigma\right\}$ be the second hierarchy LTSs of the linguistic term $s_{t}$ in $S$, and they are fully independent. A DHLTS, $s_{O}$, is in mathematical form of

$$
S_{O}=\left\{s_{t<o_{k}^{t}>} \mid t=-\tau, \ldots,-1,0,1, \ldots, \tau ; k=-\varsigma, \ldots,-1,0,1, \ldots, \varsigma\right\}
$$

we call $s_{t<o_{k}^{t}>}$ the DHLT, where $o_{k}^{t}$ expresses the second hierarchy linguistic term of the linguistic term $s_{t}$ in $S$. For convenience, a unified form $S_{O}=\left\{s_{t<o_{k}>} \mid t=-\tau, \ldots,-1,0,1, \ldots, \tau\right.$; $k=-\varsigma, \ldots,-1,0,1, \ldots, \varsigma\}$ can be used to express the DHLTS.

In recent two years, lots of studies about double hierarchy linguistic information and double hierarchy hesitant fuzzy linguistic information have been developed including preference relations (Gou et al. 2018a, 2020, 2019a, 2019b), measure methodologies (Fu \& Liao, 2019) and decision methodologies (Gou et al., 2017, 2018a, 2020; Gou \& Liao, 2019; Krishankumar et al. 2019; Liu et al., 2019; Montserrat-Adell et al., 2019), etc.

To facilitate the calculations, Gou et al. (2017) proposed two functions to make the mutual transformations between the DHLT and the numerical scale:

Definition 1.1 (Gou et al., 2017). Let $\bar{S}_{O}=\left\{s_{t<o_{k}>} \mid t \in[-\tau, \tau] ; k \in[-\varsigma, \varsigma]\right\}$ be a continuous DHLTS, Then the numerical scale $\gamma$ and the subscript $(t, k)$ of the DHLT $s_{t<o_{k}>}$ which expresses the equivalent information to the membership degree $\gamma$ can be transformed to each other by the following functions $f$ and $f^{-1}$ :

$$
f:[-\tau, \tau] \times[-\varsigma, \varsigma] \rightarrow[0,1], f(t, k)=\frac{k+(\tau+t) \varsigma}{2 \varsigma \tau}=\gamma
$$




$$
\begin{aligned}
& f^{-1}:[0,1] \rightarrow[-\tau, \tau] \times[-\varsigma, \varsigma], f^{-1}(\gamma)=[2 \tau \gamma-\tau]<o_{\varsigma(2 \tau \gamma-\tau-[2 \tau \gamma-\tau])}>= \\
& {[2 \tau \gamma-\tau]+1<o_{\varsigma((2 \tau \gamma-\tau-[2 \tau \gamma-\tau])-1)}>.}
\end{aligned}
$$

Suppose that $s_{t<o_{k}>}, s_{t^{1}<o_{k^{1}}>}$ and $s_{t^{2}<o_{k^{2}}>}$ are three DHLTs, and $\lambda(0 \leq \lambda \leq 1)$ is a real number. Then,

(1) $s_{t^{1}<o_{k^{1}}>} \oplus s_{t^{2}<o_{k^{2}}>}=s_{t^{1}+t^{2}<o_{k^{1}+k^{2}}>}$, if $t^{1}+t^{2} \leq \tau, k^{1}+k^{2} \leq \varsigma$;

(2) $\lambda s_{t<o_{k}>}=s_{\lambda t<o_{\lambda k}>}$.

In the GDM problem with double hierarchy linguistic preference environment, let $A$ and $E$ be the fixed set of alternatives and experts respectively, the experts evaluate alternatives and provide their preference information expressed by DHLPRs (Gou et al., 2017). The DHLPR is defined as follows:

Definition 1.2 (Gou et al., 2017). Let $S_{O}=\left\{s_{t<o_{k}>} \mid t=-\tau, \ldots,-1,0,1, \ldots, \tau ; k=-\varsigma, \ldots,-1,0,1, \ldots, \varsigma\right\}$ be a DHLTS. A DHLPR $R$ is presented by a matrix $R=\left(r_{i j}\right)_{m \times m} \subset A \times A$, where $r_{i j} \in S_{O}$ $(i, j=1,2, \ldots, m)$ is a DHLT, indicating the degree of the alternative $A_{i}$ over $A_{j}$. For all $i, j=1,2, \ldots, m, r_{i j}(i<j)$ satisfies the conditions $r_{i j}+r_{j i}=s_{0<o_{0}>}$ and $r_{i i}=s_{0<o_{0}>}$.

\section{Linguistic preference orderings and transformation models}

In this section, two kinds of LPOs in continuous form and in decentralized form respectively are proposed firstly. In addition, two definitions of additively consistent DHLPRs from different angles are developed, and we propose two corresponding consistency measures. Finally, two kinds of equation sets are introduced to transform LPOs into DHLPRs with complete consistencies.

\subsection{The description of two LPOs}

In the MEGM process, when experts evaluate all alternatives and provide their preference orderings, two forms of LPOs are very familiar. One is to rank all alternatives using a LPO in continuous form directly, and the other one is to give the relationship between any two alternatives and then all these relations make up a set of preference orderings. The descriptions of these two LPOs are given in detail below:

(1) The LPO in continuous form

Let $S_{O}=\left\{s_{t<o_{k}>} \mid t=-\tau, \ldots,-1,0,1, \ldots, \tau ; k=-\varsigma, \ldots,-1,0,1, \ldots, \varsigma\right\}$ be a DHLTS. Suppose that an expert $e^{a}$ provides his/her linguistic preference information on a set of alternatives $A=\left\{A_{1}, A_{2}, \ldots, A_{m}\right\}$ by a LPO denoting as:

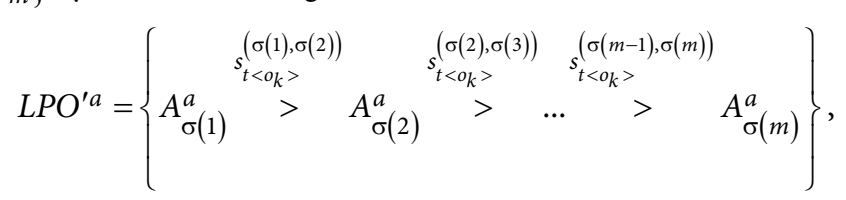

where $\bigoplus_{i=1}^{m-1} s_{t<o_{k}>}^{(\sigma(i), \sigma(i+1))} \leq s_{\tau<o_{\varsigma}>}, A_{\sigma(i)}^{a}$ denotes the $i$-th largest alternative, and the linguistic 
preference, denoted as a DHLT $s_{t<o_{k}>}^{(\sigma(i), \sigma(i+1))}$, means that the degree of the $i$-th largest alternative is better than the $i+1$-th largest alternative. Without loss of generality, the lower the position of an alternative in a LPO is, the better the alternative will be.

For example, suppose that a set of alternatives is $A=\left\{A_{1}, A_{2}, A_{3}, A_{4}\right\}$, and a LPO in continuous form may be denoted as $L P O^{\prime}=\left\{\begin{array}{lllll}\text { a little high } & \text { very much high only a little very high } \\ A_{2} & > & A_{3} & A_{1} & A_{4} \\ & & & & \end{array}\right\}$. This LPO means that the ordering of alternatives is $A_{2}>A_{3}>A_{1}>A_{4}$ and $A_{\sigma(1)}=A_{2}$, $A_{\sigma(2)}=A_{3}, A_{\sigma(3)}=A_{1}$, and $A_{\sigma(4)}=A_{4}$. Additionally, $A_{2}$ is a little higher than $A_{3}, A_{3}$ is very much higher than $A_{1}$, and $A_{1}$ is absolutely higher than $A_{4}$.

Remark 2.1. For a LPO in continuous form, considering that the evaluation $s_{t<o_{k}>}^{(\sigma(1), \sigma(m))}$ between the alternative ranked in the first position $A_{1}$ and that in the final position $A_{m}$ must be less than $s_{\tau<o_{\zeta}}$, and the sum of all evaluations in the LPO should be equal to $s_{t<o_{k}>}^{(\sigma(1), \sigma(m))}$. Therefore, we have $s_{t<o_{k}>}^{(\sigma(1), \sigma(m))}=\bigoplus_{i=1}^{m-1} s_{t<o_{k}>}^{(\sigma(i), \sigma(i+1))} \leq s_{\tau<o_{\varsigma}>}$.

(2) The LPO in decentralized form

Considering that the complexity of things and the fuzziness of people's cognition, sometimes some experts prefer to give some pairwise comparisons between any two alternatives, rather than provide a complete preference ordering. Suppose that an expert $e^{a}$ provides his preference information on a set of alternatives $A$ by a LPO in decentralized form, which is a set of preference ordering pairs and can be denoted as:

$$
L P O^{\prime \prime} a=\left\{A_{i}^{a} s_{t<o_{k}>}^{i j} A_{j}^{a} \mid s_{t<o_{k}>}^{i j} \in S_{O}, \quad i, j=1,2, \ldots, m ; i \neq j\right\},
$$

where $s_{t<o_{k}>}^{i j}$ expresses the relationship between the alternatives $A_{i}^{a}$ and $A_{j}^{a}(i, j=1,2, \ldots, m$; $i \neq j)$.

Remark 2.2. In a LPO $L P O^{\prime \prime}$, to obtain the preference information more completely and transform the LPO into DHLPR successfully and exactly, the original preference pairs should contain all alternatives and should also have some relations among alternatives directly or indirectly. Therefore, the number of the preference ordering pairs should not be less than $m-1$. For example, suppose that a set of alternatives $A=\left\{A_{1}, A_{2}, A_{3}, A_{4}\right\}$, and an expert provides his/her LPO in decentralized form, which is denoted as

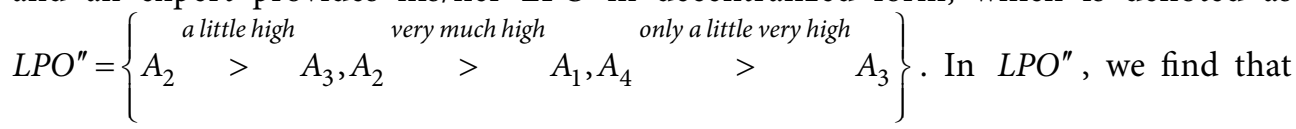

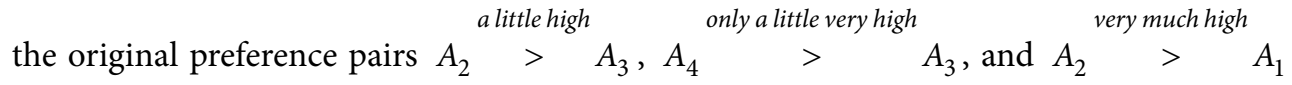
have relationships directly. However, the relationships between $A_{1}$ and $A_{3}$, between $A_{1}$ and $A_{4}$, and between $A_{2}$ and $A_{4}$ are indirectly, which can be obtained by a method proposed in Subsection 2.3. 


\subsection{The consistency measure of DHLPR}

As we discussed in Introduction, it is necessary to transform all LPOs into DHLPRs and obtain the uniformed information when the original linguistic preferences are represented by LPOs. As we know, the most important and primary work is to ensure that all DHLPRs are consistent before making a decision with DHLPRs. Therefore, this paper will develop transformation models which can obtain the DHLPRs with acceptable additive consistencies. Before establishing the transformation models, the definition of additive consistent DHLPR should be proposed firstly.

Definition 2.1. A DHLPR $R=\left(r_{i j}\right)_{m \times m} \subset A \times A$ can be called an additively consistent DHL$\mathrm{PR}$ if it satisfies

$$
r_{i j}=r_{i \rho}+r_{\rho j} \quad(i, j, \rho=1,2, \ldots, m, i \neq j) .
$$

Based on Definition 3.1, a theorem can be developed as follows:

Theorem 2.1. Let $R=\left(r_{i j}\right)_{m \times m} \subset A \times A$ be a DHLPR. If $\overline{r_{i j}}=\frac{1}{m}\left(\underset{\rho=1}{\oplus}\left(r_{i \rho}+r_{\rho j}\right)\right)$ for $i, j, \rho=$ $1,2, \ldots, m, i \neq j$, then $\bar{R}=\left(\bar{r}_{i j}\right)_{m \times m} \subset A \times A$ is an additively consistent DHLPR.

Additionally, based on the transformation function $f$, the definition of an additive consistency for a DHLPR also can be developed as follows:

Definition 2.2. A DHLPR $R=\left(r_{i j}\right)_{m \times m} \subset A \times A$ can be called an additively consistent DHLPR if it satisfies

$$
f\left(r_{i j}\right)=f\left(r_{i \rho}\right)+f\left(r_{\rho j}\right)-0.5 \quad(i, j, \rho=1,2, \ldots, m, i \neq j) .
$$

Similarly, a theorem can be obtained as follows:

Theorem 2.2. Let $R=\left(r_{i j}\right)_{m \times m} \subset A \times A$ be a DHLPR. If $f\left(\bar{r}_{i j}\right)=\frac{1}{m}\left(\underset{\rho=1}{\oplus}\left(f\left(r_{i \rho}\right)+f\left(r_{\rho j}\right)-0.5\right)\right)$ for $i, j, \rho=1,2, \ldots, m, i \neq j$, then $\bar{R}=\left(\bar{r}_{i j}\right)_{m \times m} \subset A \times A$ is an additively consistent DHLPR.

In actual decision making process, it is very important to check whether a DHLPR is with acceptable consistency. Based on Theorem 3.2, a consistency index (CI) for a DHLPR is defined as follows:

Definition 2.3. Let $R=\left(r_{i j}\right)_{m \times m} \subset A \times A$ be a DHLPR. Then the consistency index for $R$ is obtained as:

$$
C I(R)=1-\frac{2}{3 m(m-1)(m-2)} \sum_{i=1}^{m-1} \sum_{j=1, j \neq i}^{m} \sum_{\rho=1, \rho \neq i, j}^{m}\left|f\left(r_{i j}\right)-f\left(r_{i \rho}\right)-f\left(r_{\rho j}\right)+0.5\right| .
$$

Obviously, $C I(R)=1$ means that $f\left(r_{i j}\right)=f\left(r_{i \rho}\right)+f\left(r_{\rho j}\right)-0.5$, which is equivalent to $f\left(r_{i j}\right)-0.5=\left(f\left(r_{i \rho}\right)-0.5\right)+\left(f\left(r_{\rho j}\right)-0.5\right)$, and this situation is called additive transitivity or additive consistency (Herrera-Viedma et al., 2004).

Additionally, there exists a mass of redundant computations in Eq. (8). For example, let $\delta_{i j \rho}=\left|f\left(r_{i j}\right)-f\left(r_{i \rho}\right)-f\left(r_{\rho j}\right)+0.5\right|$, then we can obtain that $\delta_{i j \rho}=\delta_{i \rho j}=\delta_{j i \rho}=\delta_{j \rho i}=\delta_{\rho i j}=\delta_{\rho j i}$. Therefore, Eq. (8) can be rewritten as: 


$$
\begin{aligned}
& C I(R)=1-\frac{4}{m(m-1)(m-2)} \sum_{1 \leq i<j<\rho \leq m}^{m-1}\left|f\left(r_{i j}\right)-f\left(r_{i \rho}\right)-f\left(r_{\rho j}\right)+0.5\right|= \\
& 1-\frac{4}{m(m-1)(m-2)} \sum_{i=1}^{m-2} \sum_{j=i+1}^{m-1} \sum_{\rho=j+1}^{m}\left|f\left(r_{i j}\right)-f\left(r_{i \rho}\right)-f\left(r_{\rho j}\right)+0.5\right| .
\end{aligned}
$$

Based on Eq. (7), the DHLPR $\mathbb{R}$ is completely consistent when $C I(R)=1$. In actual decision-making process, however, it is very difficult that the DHLPRs provided by experts are completely consistent. Therefore, setting a consistency threshold, denoted by $\overline{C I}$, is necessary in the beginning of decision-making (Dong et al., 2008; Gou et al., 2020, 2019b). In this paper, we refer to the consistency thresholds developed by Gou, Liao, Wang, Xu, and Herrera (2020), which are listed in Table 1 where $m$ is the number of alternatives and $T$ is the number of linguistic terms in the first hierarchy LTS.

Table 1. The values of consistency thresholds $\overline{C I}$ based on different $m$ and $T$

\begin{tabular}{|c|c|c|c|c|c|c|}
\hline & $m=3$ & $m=4$ & $m=5$ & $m=6$ & $m=7$ & $m=8$ \\
\hline$T=5$ & 0.8793 & 0.6970 & 0.6512 & 0.6226 & 0.6030 & 0.5888 \\
\hline$T=9$ & 0.8897 & 0.8485 & 0.8256 & 0.8113 & 0.8015 & 0.7944 \\
\hline$T=17$ & 0.9448 & 0.9242 & 0.9128 & 0.9056 & 0.9007 & 0.8972 \\
\hline
\end{tabular}

If $C I(R)<\overline{C I}$, then the experts need to revise their preference information according to the feedback.

\subsection{The transformation models for transforming LPOs into DHLPRs}

Based on these two kinds of LPOs discussed in Subsection 3.1, we can develop two transformation models to transform LPOs into DHLPRs on the basis of the additively consistent DHLPRs, respectively.

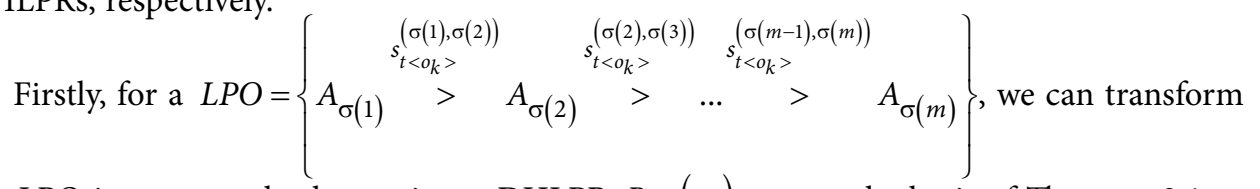
the LPO into a completely consistent DHLPR $R=\left(r_{i j}\right)_{m \times m}$ on the basis of Theorem 2.1 or Theorem 2.2.

To get al. preference information of a DHLPR, we only need to calculate the elements of the upper triangular matrix. For a DHLPR $R=\left(r_{i j}\right)_{m \times m}$, the elements of main diagonal is $r_{i i}=s_{0<o_{0}>}(i=1,2, \ldots, m)$. Additionally, we can also obtain the elements of $r_{\sigma(i) \sigma(i+1)}=s_{t<o_{k}>}^{(\sigma(i), \sigma(i+1))}(i=1,2, \ldots, m-1)$ from the given LPO directly. Then we can only need to calculate the remaining elements of the upper triangular matrix and the number of them is $(m-1)(m-2) / 2$.

Based on Definition 2.1 and Theorem 2.1, we develop an equation set including $(m-1)(m-2) / 2$ equations, which can be used to obtain the remaining elements of the upper triangular matrix: 


$$
\left\{\begin{array}{l}
r_{\sigma(1), \sigma(3)}=\frac{1}{m}\left(\bigoplus_{\rho=1}^{m}\left(r_{\sigma(1), \rho}+r_{\rho, \sigma(3)}\right)\right) ; \ldots ; r_{\sigma(1), \sigma(m)}=\frac{1}{m}\left(\bigoplus_{\rho=1}^{m}\left(r_{\sigma(1), \rho}+r_{\rho, \sigma(m)}\right)\right), \\
r_{\sigma(2), \sigma(4)}=\frac{1}{m}\left(\bigoplus_{\rho=1}^{m}\left(r_{\sigma(2), \rho}+r_{\rho, \sigma(4)}\right)\right) ; \ldots ; r_{\sigma(2), \sigma(m)}=\frac{1}{m}\left(\bigoplus_{\rho=1}^{m}\left(r_{\sigma(2), \rho}+r_{\rho, \sigma(m)}\right)\right), \\
\ldots \\
r_{\sigma(m-2), \sigma(m)}=\frac{1}{m}\left(\stackrel{m}{\oplus}\left(r_{\sigma(m-2), \rho}+r_{\rho, \sigma(m)}\right)\right) .
\end{array}\right.
$$

Similarly, based on Definition 2.2 and Theorem 2.2, the other kind of equation set can be developed to obtain the remaining elements of the upper triangular matrix:

$$
\left\{\begin{array}{l}
f\left(r_{\sigma(1) \sigma(3)}\right)=\frac{1}{m}\left(\bigoplus_{\rho=1}^{\oplus}\left(f\left(r_{\sigma(1) \rho}\right)+f\left(r_{\rho \sigma(3)}\right)-0.5\right)\right) ; \ldots ; f\left(r_{\sigma(1) \sigma(m)}\right)=\frac{1}{m}\left(\bigoplus_{\rho=1}^{m}\left(f\left(r_{\sigma(1) \rho}\right)+f\left(r_{\rho \sigma(m)}\right)-0.5\right)\right), \\
f\left(r_{\sigma(2) \sigma(4)}\right)=\frac{1}{m}\left(\oplus_{\rho=1}^{m}\left(f\left(r_{\sigma(2) \rho}\right)+f\left(r_{\rho \sigma(4)}\right)-0.5\right)\right) ; \ldots ; f\left(r_{\sigma(2) \sigma(m)}\right)=\frac{1}{m}\left(\oplus_{\rho=1}^{m}\left(f\left(r_{\sigma(2) \rho}\right)+f\left(r_{\rho \sigma(m)}\right)-0.5\right)\right), \\
\ldots \\
f\left(r_{\sigma(m-2) \sigma(m)}\right)=\frac{1}{m}\left(\bigoplus_{\rho=1}^{m}\left(f\left(r_{\sigma(m-2) \rho}\right)+f\left(r_{\rho \sigma(m)}\right)-0.5\right)\right) .
\end{array}\right.
$$

Obviously, in both models (10) and (11), some repeating elements $r_{i i}(i=1,2, \ldots, m)$ exist in every equation. Therefore, the models (10) and (11) are rewritten as follows:

$$
\begin{aligned}
& r_{\sigma(1) \sigma(3)}=\frac{1}{m-2}\left(\underset{\rho=1, \rho \neq \sigma(1), \rho \neq \sigma(3)}{\stackrel{m}{\oplus}}\left(r_{\sigma(1) \rho}+r_{\rho \sigma(3)}\right)\right) ; \ldots ; r_{\sigma(1) \sigma(m)}=\frac{1}{m-2}\left(\underset{\rho=1, \rho \neq \sigma(1), \rho \neq \sigma(m)}{\stackrel{m}{\oplus}}\left(r_{\sigma(1) \rho}+r_{\rho \sigma(m)}\right)\right), \\
& \left\{r_{\sigma(2) \sigma(4)}=\frac{1}{m-2}\left(\underset{\rho=1, \rho \neq \sigma(2), \rho \neq \sigma(4)}{\stackrel{m}{\oplus}}\left(r_{\sigma(2) \rho}+r_{\rho \sigma(4)}\right)\right) ; \ldots ; r_{\sigma(2) \sigma(m)}=\frac{1}{m-2}\left(\underset{\rho=1, \rho \neq \sigma(2), \rho \neq \sigma(m)}{\stackrel{m}{\oplus}}\left(r_{\sigma(2) \rho}+r_{\rho \sigma(m)}\right)\right)\right. \text {, } \\
& \text {... } \\
& r_{\sigma(m-2) \sigma(m)}=\frac{1}{m-2}\left(\stackrel{m}{\stackrel{m}{\oplus=1, \rho \neq \sigma(m-2), \rho \neq \sigma(m)}}\left(r_{\sigma(m-2) \rho}+r_{\rho \sigma(m)}\right)\right) ;
\end{aligned}
$$

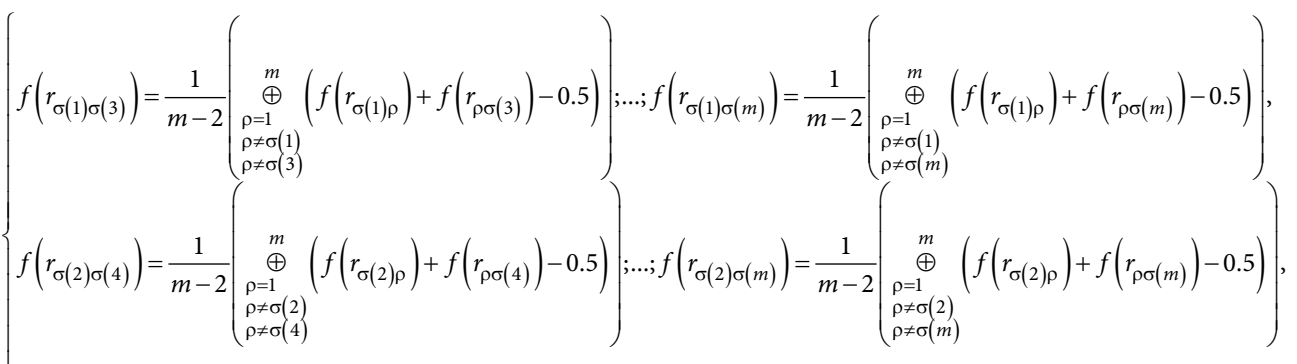$$
f\left(r_{\sigma(m-2) \sigma(m)}\right)=\frac{1}{m-2}\left(\underset{\rho=1, \rho \neq \sigma(m-2), \rho \neq \sigma(m)}{\stackrel{m}{\oplus}}\left(f\left(r_{\sigma(m-2) \rho}\right)+f\left(r_{\rho \sigma(m)}\right)-0.5\right)\right) .
$$

Remark 2.3. When we transform the second kind of LPO in decentralized form, the fundamental is also to calculate the unknown elements with the existing linguistic preference information. Therefore, we can also utilize the model (12) or (13) to obtain the remaining elements of a DHLPR. Additionally, considering that the DHLPR is developed based on Theorems 2.1 and 2.2, so the obtained DHLPR $\mathbb{R}$ is completely consistent. 
Two examples can be set up to show the process of transformation models clearly:

$\underset{c}{\text { Example 2.1. Let }} \begin{aligned} & s_{1<o_{2}>} \\ & s_{0<o_{1}>}>\end{aligned} S_{O}=\left\{s_{t<o_{k}>} \mid t=-4, \ldots, 4 ; k=-4, \ldots, 4\right\}$ be a DHLTS, $L P O^{\prime}=\left\{\begin{array}{c}s_{2<o_{1}>} \\ A_{2}>\end{array}\right.$ $A_{3}>A_{1}>A_{4}$
as:

$$
R^{\prime}=\left(r_{i j}\right)_{4 \times 4}=\left(\begin{array}{cccc}
s_{0<o_{0}>} & - & s_{-1<o_{-2}>} & s_{0<o_{1}>} \\
- & s_{0<o_{0}>} & s_{2<o_{1}>} & - \\
s_{1<o_{2}>} & s_{-2<o_{-1}>} & s_{0<o_{0}>} & - \\
s_{0<o_{-1}>} & - & - & s_{0<o_{0}>}
\end{array}\right) .
$$

Therefore, it is only necessary to calculate $r_{12}, r_{24}$, and $r_{34}$. Based on the model (12) or (13), two kinds of equation sets are listed as follows:

$$
\left\{\begin{array}{l}
f\left(r_{12}\right)=\frac{1}{2}\left(\underset{\rho=3, \rho=4}{\oplus}\left(f\left(r_{1 \rho}\right)+f\left(r_{\rho 2}\right)-0.5\right)\right) \\
f\left(r_{24}\right)=\frac{1}{2}\left(\underset{\rho=1, \rho=3}{\oplus}\left(f\left(r_{2 \rho}\right)+f\left(r_{\rho 4}\right)-0.5\right)\right) \\
f\left(r_{34}\right)=\frac{1}{2}\left(\underset{\rho=1, \rho=2}{\oplus}\left(f\left(r_{3 \rho}\right)+f\left(r_{\rho 4}\right)-0.5\right)\right)
\end{array}\right)\left\{\begin{array}{l}
r_{12}=\frac{1}{2}\left(\underset{\rho=3, \rho=4}{\oplus}\left(r_{1 \rho}+r_{\rho 2}\right)\right) \\
r_{24}=\frac{1}{2}\left(\underset{\rho=1, \rho=3}{\oplus}\left(r_{2 \rho}+r_{\rho 4}\right)\right) \\
r_{34}=\frac{1}{2}\left(\underset{\rho=1, \rho=2}{\oplus}\left(r_{3 \rho}+r_{\rho 4}\right)\right)
\end{array} .\right.
$$

Solving these two equation sets, we obtain $r_{12}=s_{-4<o_{2}>}, r_{24}=s_{4<o_{0}>}$, and $r_{34}=s_{1<o_{2}>}$. Then, the consistent DHLPR is obtained as:

$$
\bar{R}^{\prime}=\left(\begin{array}{cccc}
s_{0<o_{0}>} & s_{-4<o_{1}>} & s_{-1<o_{-2}>} & s_{0<o_{1}>} \\
s_{4<o_{-1}>} & s_{0<o_{0}>} & s_{2<o_{1}>} & s_{4<o_{0}>} \\
s_{1<o_{2}>} & s_{-2<o_{-1}>} & s_{0<o_{0}>} & s_{1<o_{3}>} \\
s_{0<o_{-1}>} & s_{-4<o_{0}>} & s_{-1<o_{-3}>}> & s_{0<o_{0}>}
\end{array}\right) .
$$

Example 2.2. Let $S_{O}=\left\{s_{t<o_{k}>} \mid t=-4, \ldots, 4 ; k=-4, \ldots, 4\right\}$ be a DHLTS, LPO" $=\left\{\begin{array}{c}s_{1<o_{1}>} \\ A_{2}>\end{array}\right.$ $\left.A_{3}, A_{2} \stackrel{s_{2<o_{1}>}>}{>} A_{1}, A_{1} \stackrel{s_{1<o_{-1}>}}{>} A_{4}\right\}$ be a LPO. Then the incomplete DHLPR, denoted by $R^{\prime \prime}$, is established as:

$$
R^{\prime \prime}=\left(r_{i j}\right)_{4 \times 4}=\left(\begin{array}{cccc}
s_{0<o_{0}>} & s_{-2<o_{-1}>} & - & s_{1<o_{-1}>} \\
s_{2<o_{1}>} & s_{0<o_{0}>} & s_{1<o_{1}>} & - \\
- & s_{-1<o_{-1}>} & s_{0<o_{0}>} & - \\
s_{-1<o_{1}>} & - & - & s_{0<o_{0}>}
\end{array}\right) .
$$

Similarly, we need to calculate $r_{13}, r_{24}$, and $r_{34}$ based on Eq. (12) or (13), and two kinds of the equation sets are listed as follows:

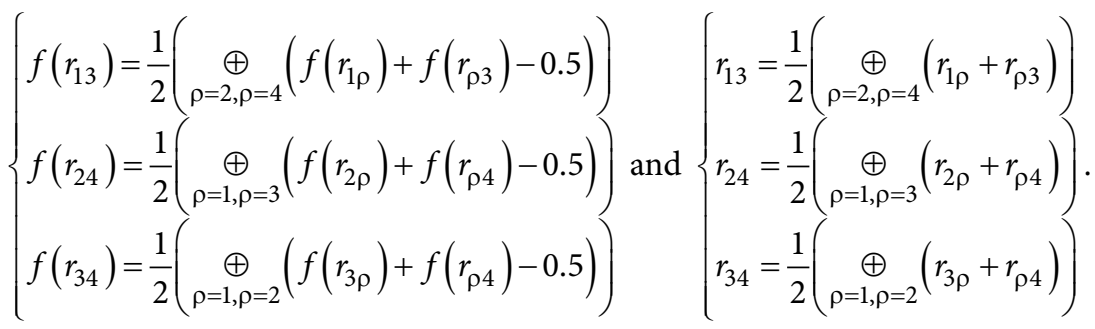


Solving these two equation sets, we obtain $r_{13}=s_{-1<o_{0}>}, r_{24}=s_{3<o_{0}>}$, and $r_{34}=s_{1<o_{3}>}$. Then, the consistent DHLPR is obtained as:

$$
\bar{R}^{\prime \prime}=\left(r_{i j}\right)_{4 \times 4}=\left(\begin{array}{cccc}
s_{0<o_{0}>} & s_{-2<o_{-1}>} & s_{-1<o_{0}>} & s_{1<o_{-1}>} \\
s_{2<o_{1}>} & s_{0<o_{0}>} & s_{1<o_{1}>} & s_{3<o_{0}>} \\
s_{1<o_{0}>} & s_{-1<o_{-1}>} & s_{0<o_{0}>} & s_{1<o_{3}>} \\
s_{-1<o_{1}>} & s_{-3<o_{0}>} & s_{-1<o_{-3}>} & s_{0<o_{0}>}
\end{array}\right) .
$$

\section{The consensus model for LPOs}

A MEDM problem with linguistic preference information can be described as follows: $A=\left\{A_{1}, A_{2}, \ldots, A_{m}\right\}$ is a set of alternatives, $E=\left\{e^{1}, e^{2}, \ldots, e^{n}\right\}$ is a set of experts, and experts' preference information is expressed by some LPOs $\left\{L P O^{1}, L P O^{2}, \ldots, L P O^{n}\right\}$.

After obtaining the DHLPRs of all experts with the completely acceptable consistencies, the next step will focus on reaching group consensus by developing a consensus reaching method. Therefore, this section will introduce a multi-stage consensus optimization model to reach group consensus firstly, and then an interactive consensus reaching algorithm is proposed on the basis of the proposed consensus model.

\subsection{Group consensus measures}

This subsection mainly proposes two group consensus measures to calculate the consensus degrees based on the distance between an individual DHLPR and the collective DHLPR, and the distance between an individual DHLPR and the remaining DHLPRs, respectively.

Firstly, the collective DHLPRs can be obtained by the following aggregating method:

Definition 3.1. Let $\left\{R_{1}, R_{2}, \ldots, R_{n}\right\}$ be a set of individual DHLPRs, where $R_{a}=\left(r_{i j}^{a}\right)_{m \times m}$

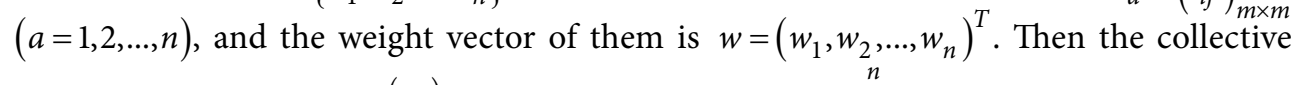
DHLPR, denoted by $R_{c}=\left(r_{i j}^{c}\right)_{m \times m}$, is obtained, where $r_{i j}^{c}=\bigoplus_{a=1}^{n} w_{a} r_{i j}^{a}$.

Then the consensus degree of an individual DHLPR is defined as follows:

Definition 3.2. Let $\left\{R_{1}, R_{2}, \ldots, R_{n}\right\}$ be a set of individual DHLPRs transformed from the LPOs provided by the experts $e^{a}(a=1,2, \ldots, n)$, where $R_{a}=\left(r_{i j}^{a}\right)_{m \times m}, R_{c}=\left(r_{i j}^{c}\right)_{m \times m}$ be a collective DHLPR. Then, the consensus degree of an individual DHLPR $R_{a}$ is calculated by

$$
C D^{\prime}\left(R_{a}\right)=1-d\left(R_{a}, R_{c}\right)=1-\frac{2}{m(m-1)} \sum_{i=1}^{m-1} \sum_{j=i+1}^{m}\left|f\left(r_{i j}^{a}\right)-f\left(r_{i j}^{c}\right)\right| .
$$

Additionally, the group consensus degree is obtained by

$$
C D^{\prime}=\min _{a} C D^{\prime}\left(R_{a}\right) \text {. }
$$

Suppose that $\overline{C D}$ is a given consensus threshold, then the group consensus has been reached if the group consensus degree satisfies

$$
C D^{\prime}=\min _{a} C D^{\prime}\left(R_{a}\right) \geq \overline{C D} .
$$


Additionally, if we only consider the distance between any two different DHLPRs instead of the distance between an individual and collective DHLPRs, the other consensus degree of a DHLPR is defined as follows:

Definition 3.3. Let $\left\{R_{1}, R_{2}, \ldots, R_{n}\right\}$ be a set of individual DHLPRs transformed from the LPOs provided by the experts $e^{a}(a=1,2, \ldots, n)$, and $R_{a}=\left(r_{i j}^{a}\right)_{m \times m}$. Then, the consensus degree for an individual DHLPR $\mathbb{R}_{a}$ is calculated by

$C D^{\prime \prime}\left(R_{a}\right)=1-\frac{1}{n-1} \sum_{b=1 ; b \neq a}^{n} d\left(R_{a}, R_{b}\right)=1-\frac{2}{m(m-1)(n-1)} \sum_{b=1 ; b \neq a}^{n} \sum_{i=1}^{m-1} \sum_{j=i+1}^{m}\left|f\left(r_{i j}^{a}\right)-f\left(r_{i j}^{b}\right)\right|$.

Similarly, based on Eq. (17), the other kind of group consensus degree is obtained by

$$
C D^{\prime \prime}=\min _{a} C D^{\prime \prime}\left(R_{a}\right) \text {. }
$$

Then, the group consensus has been reached if

$$
C D^{\prime \prime}=\min _{a} C D^{\prime \prime}\left(R_{a}\right) \geq \overline{C D} .
$$

Remark 3.1. Obviously, both of two types of consensus measures are very closely related to each other. However, Definition 3.3 has more restrictive than Definition 3.2. Therefore, this paper mainly uses the second group consensus degree $C D^{\prime \prime}$ in the consensus reaching process.

Additionally, the given consensus threshold value $\overline{C D}$ can be used to decide whether the consensus reaching process can be carried out. We usually set the consensus threshold to be smaller than 0.9 (Herrera-Viedma et al., 2005; Parreiras et al., 2012). If $C D \geq \overline{C D}$, then the consensus degree of all DMs is sufficiently high and the consensus reaching process is over. Otherwise, we should make some changes about preference to improve the consensus degree and reach the given consensus threshold value. Furthermore, according to the practical situation of the decision-making problem, the value of the consensus threshold $\overline{C D}$ can be adjusted. If the demand for consensus is strict, then the threshold should be given a higher value; Otherwise, a lower threshold value should be provided.

\subsection{Multi-stage consensus optimization model}

Considering that all the transformed DHLPRs are completely consistent, it is not necessary to improve the consistencies of them before starting the consensus reaching process. This subsection develops a multi-stage consensus optimization model which can not only reach group consensus but also maintain the consistency of every DHLPR. This multi-stage optimization model consists of three objectives, the first one is to minimize the deviations of the modification magnitudes, the second one is to minimize the cardinal number of modifications while keeping the value of the first objective constant, and the third one is to minimize the number of experts who need to change their evaluations.

\section{Stage 1. minimizing the deviations of the modification magnitudes}

In general, the experts more prefer to keep their preferences constant or only want to change their preferences as little as possible. Therefore, the first stage of the multi-stage consensus 
optimization model is to make the original DHLPR and the adjusted DHLPR as close as possible. According to this, we can develop a model to minimize the deviations of the modification magnitudes. Let $R_{a}=\left(r_{i j}^{a}\right)_{m \times m}(a=1,2, \ldots, n)$ be the transformed DHLPRs provided by the experts $e^{a}(a=1,2, \ldots, n)$, and $R_{a}^{*}=\left(r_{i j}^{* a}\right)_{m \times m}(a=1,2, \ldots, n)$ be the corresponding adjusted DHLPR. Then the objective can be given as:

$$
\sum_{a=1}^{n} d\left(R_{a}, R_{a}^{*}\right)=\sum_{a=1}^{n} \sum_{i=1}^{m-1} \sum_{j=i+1}^{m}\left|f\left(r_{i j}^{a}\right)-f\left(r_{i j}^{*}\right)\right| .
$$

Due to the fact that all individual DHLPRs should be with acceptable consistencies and the group consensus also needs to be reached, then the model in this stage is developed as follows:

$$
\begin{aligned}
& \min G_{1}=\sum_{a=1}^{n} d\left(R_{a}, R_{a}^{*}\right)=\sum_{a=1}^{n} \sum_{i=1}^{m-1} \sum_{j=i+1}^{m}\left|f\left(r_{i j}^{a}\right)-f\left(r_{i j}^{* a}\right)\right| \\
& \text { s.t. }\left\{\begin{array}{lcc}
C I\left(R_{a}^{*}\right) \geq \overline{C I}, & a=1,2, \ldots, n, & (21-1) . \\
C D\left(R_{a}\right) \geq \overline{C D}, & a=1,2, \ldots, n, & (21-2) \\
r_{i j}^{*} \in \bar{S}_{O} \text { or } r_{i j}^{*} a \in S_{O}, & i<j, a=1,2, \ldots, n, & (21-3)
\end{array}\right.
\end{aligned}
$$

Based on Eq. (9) and Eq. (17), the model (21) can be changed into $\min G_{1}=\sum_{a=1}^{n} d\left(R_{a}, R_{a}^{*}\right)=\sum_{a=1}^{n} \sum_{i=1}^{m-1} \sum_{j=i+1}^{m}\left|f\left(r_{i j}^{a}\right)-f\left(r_{i j}^{*}\right)\right|$

$$
\begin{aligned}
& \text { s. }\left\{\begin{array}{ll}
\sum_{i=1}^{m-2} \sum_{j=i+1}^{m-1} \sum_{\rho=j+1}^{m}\left|f\left(r_{i j}^{* a}\right)-f\left(r_{i \rho}^{* a}\right)+f\left(r_{j \rho}^{* a}\right)-0.5\right| \leq S C I, & a=1,2, \ldots, n, \quad(22-1) \\
\sum_{\substack{n=1 ; b \neq a \\
r_{i j}^{*} a}}^{m-1} \sum_{i=1}^{m-1} \sum_{j=i+1}^{m}\left|f\left(r_{i j}^{a}\right)-f\left(r_{i j}^{*}\right)\right| \leq S C D, & a=1,2, \ldots, n, \quad(22-2)
\end{array},\right. \\
& \text { where } S C I=(1-\overline{C I}) \times \frac{m(m-1)(m-2)}{4} \text { and } S C D=(1-\overline{C D}) \times \frac{m(m-1)(n-1)}{2} \text {. }
\end{aligned}
$$

By solving Eq. (21) or (22), we can obtain at least one optimal solution, which is denoted as $G_{1}^{*}$.

\section{Stage 2. minimizing the cardinal number of modifications}

In the consensus reaching process, lots of existing methods need to change all the original preferences such as the automatic adjustment method (Gou et al., 2018a; Liao et al. 2016; Zhang \& Chen, 2019). However, it will create a huge amount of work and make the consensus process complicated. Therefore, this stage focuses on developing a model to minimize the number of modifications and keep the optimal solution obtained in first stage.

Then, we need to verify whether the original preference changes, so a constraint can be set up: 


$$
\left|f\left(r_{i j}^{a}\right)-f\left(r_{i j}^{* a}\right)\right| \leq \Im \phi_{i j}^{a},
$$

where $\mathfrak{I}$ is a sufficiently large number and $\phi_{i j}^{a} \in\{0,1\}$. Clearly, if $\left|f\left(r_{i j}^{a}\right)-f\left(r_{i j}^{* a}\right)\right|=0$ or $\phi_{i j}^{a}=0$, then the original preference $r_{i j}^{a}$ has not been changed; otherwise, the original preference $r_{i j}^{a}$ has been changed.

Based on the optimal solution $G_{1}^{*}$, the model of the second stage is developed as follows:

$$
\begin{aligned}
& \min G_{2}=\sum_{a=1}^{n} \sum_{i=1}^{m-1} \sum_{j=i+1}^{m} \phi_{i j}^{a}
\end{aligned}
$$

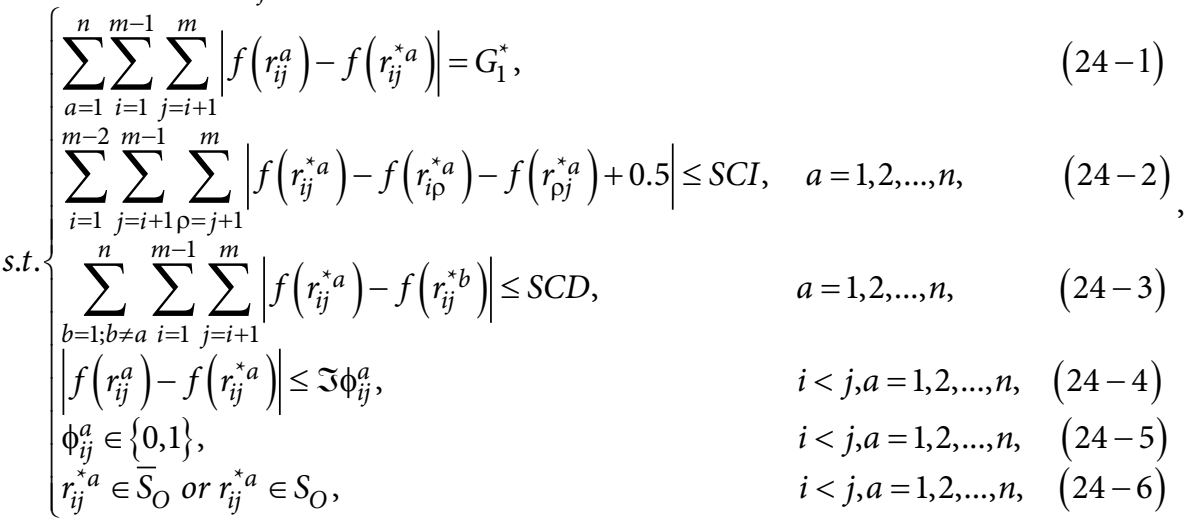

where $S C I=(1-\overline{C I}) \times \frac{m(m-1)(m-2)}{4}, S C D=(1-\overline{C D}) \times \frac{m(m-1)(n-1)}{2}$, and $\mathfrak{I}$ is a sufficiently large number.

Similarly, solving Eq. (24), we can also obtain at least one optimal solution, which is denoted as $G_{2}^{*}$.

\section{Stage 3. minimizing the number of experts who need to change their preferences}

Considering that the consensus will be reached faster if we can minimize the number of experts who need to change their preferences within reasonable limits, so this stage mainly focuses on this goal. To verify whether an expert $e^{a}$ changes his/her preference information, a constraint can be set up

$$
\sum_{i=1}^{m-1} \sum_{j=i+1}^{m}\left|f\left(r_{i j}^{a}\right)-f\left(r_{i j}^{*}\right)\right| \leq \mathfrak{I} \varphi^{a}
$$

where $\mathfrak{I}$ is a sufficiently large number and $\varphi^{a} \in\{0,1\}$. Therefore, if $\sum_{i=1}^{m-1} \sum_{j=i+1}^{m}\left|f\left(r_{i j}^{a}\right)-f\left(r_{i j}^{* a}\right)\right|=0$ or $\varphi^{z}=0$, then the expert $e^{a}$ does not change his preference information; otherwise the expert $e^{a}$ has changed some preference information.

Based on the optimal solution $G_{2}^{*}$ obtained in Eq. (24), the model of the third stage can be established as follows: 


$$
\begin{aligned}
& \min G_{3}=\sum_{a=1}^{n} \varphi^{a}
\end{aligned}
$$

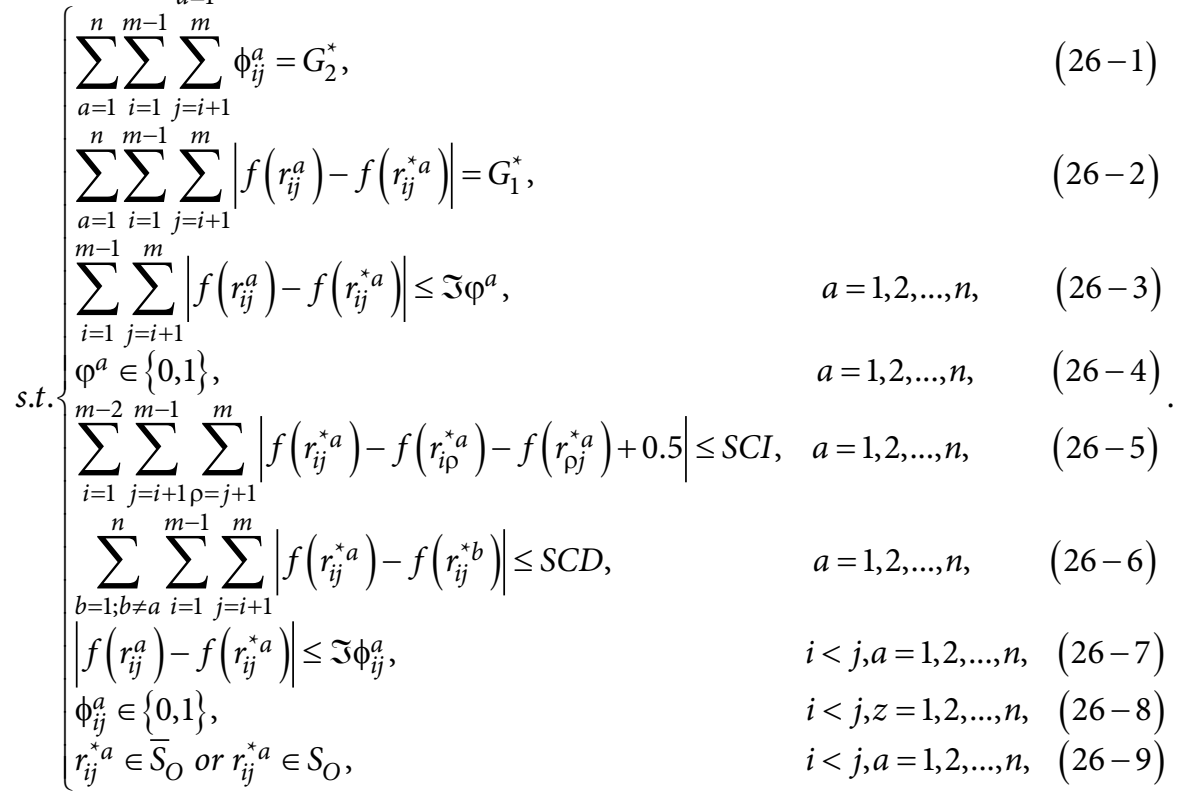

Similarly, solving the model (26), at least one optimal solution can be obtained, denoted as $G_{3}^{*}$.

When we obtain the optimal adjusted DHLPRs $R_{a}^{*}=\left(r_{i j}^{*} a\right)_{m \times m}(a=1,2, \ldots, n)$, the next step is to identify the set of experts who should change their preferences. Moreover, it is necessary to identify the positions that should be changed for each expert $e^{a}$. The identification rules can be developed as follows:

(1) Based on the optimal solutions obtained in the model (26), the set of experts who need to change their preferences can be obtained:

$$
E S=\left\{e^{a}\left|\sum_{i=1}^{m-1} \sum_{j=i+1}^{m}\right| f\left(r_{i j}^{a}\right)-f\left(r_{i j}^{*} a\right) \mid \neq 0, a=1,2, \ldots, n\right\} .
$$

(2) For every expert $e^{a} \in E S$, we have

$$
P\left(e^{a}\right)=\left\{(i, j)|| f\left(r_{i j}^{a}\right)-f\left(r_{i j}^{*} a\right) \mid>0, i, j=1,2, \ldots, m, i<j\right\},
$$

where the positions $(i, j)(i, j=1,2, \ldots, m, i<j)$ that should be changed for the expert $e^{a}$ can be identified.

Remark 3.2. After identifying the expert $e^{a} \in E S$ and the positions $(i, j)$ by Eq. (27) and Eq. (28), we need to feedback the adjustment suggestions to the corresponding experts. In this process, an interval value can be developed as follows:

$$
I V=\left[\min \left\{r_{i j}^{a}, r_{i j}^{* a}\right\}, \max \left\{r_{i j}^{a}, r_{i j}^{* a}\right\}\right], \quad a=1,2, \ldots, n .
$$

Then, the experts can provide their adjusted preferences according to the suggested interval values. 


\subsection{An interactive consensus reaching algorithm with LPOs}

This section mainly develops an interactive consensus reaching algorithm with LPOs in MEDM. This algorithm consists of two parts. One is to transform the LPOs into the corresponding DHLPRs $R_{a}=\left(r_{i j}^{a}\right)_{m \times m}(a=1,2, \ldots, n)$ with complete consistencies, and we do not need to check the consistency of each DHLPR anymore. The other one is to reach group consensus on the basis of the multi-stage consensus optimization model discussed in Subsection 3.2.

The consensus reaching process is a dynamic decision-making process. Firstly, based on Definition 3.2, we can obtain the consensus degree $C D^{\prime \prime}\left(R_{a}\right)$ of each DHLPR and decide which one needs to adjust his/her preference. Secondly, we need to use the multi-stage consensus optimization model to obtain the optimal solution. In this process, we can develop an identification rules which can identify the expert who has to adjust his preference and the positions that should be adjusted. Additionally, we can also get the suggestions by combining the original DHLPR and the optimal solution, and then feedback the suggestions to the expert. Finally, we update the preference based on the adjustments provided by the expert.

Algorithm 1. A multi-stage interactive consensus reaching algorithm with LPOs

Input: The original LPOs $\left\{L P O^{1}, L P O^{2}, \ldots, L P O^{n}\right\}$, the given consistency threshold value $\overline{C I}$, the given consensus threshold value $\overline{C D}$, and the maximum iteration number $Z_{\max }$.

Output: The improved DHLPRs $\left\{{ }^{\star} R_{1},{ }^{\star} R_{2}, \ldots,{ }^{\star} R_{n}\right\}$, the final reached consensus degree for each expert $e^{a}, C D\left({ }^{\star} R_{a}\right)(a=1,2, \ldots, n)$, and the iteration number $Z_{a}(z=1,2, \ldots, n)$.

Step 1. Based on Eq. (12) or (13), we transform each LPO to the corresponding DHLPR with complete consistency, $R_{a}=\left(r_{i j}^{a}\right)_{m \times m}(a=1,2, \ldots, n)$.

Step 2. Let $Z=1$, and $R_{a}^{(Z)}=\left(r_{i j}^{a,(Z)}\right)_{m \times m}=\left(r_{i j}^{a}\right)_{m \times m}(a=1,2, \ldots, n)$.

Step 3. Based on Eq. (17), we obtain the consensus degree for each DHLPR $R_{a}^{(Z)}, C D^{\prime \prime}\left(R_{a}^{(Z)}\right)$ $(a=1,2, \ldots, n)$. If $C D^{\prime \prime}\left(R_{a}^{(Z)}\right) \geq \overline{C D}$ or $Z>Z_{\max }$, then go to Step 6; otherwise, go to Step 4 . Step 4. Calculate the optimal adjusted DHLPR $R_{a}^{*}(Z)=\left(r_{i j}^{* a,(Z)}\right)_{m \times m}(a=1,2, \ldots, n)$ on the basis of the multi-stage consensus optimization models proposed in Subsection 4.2.

Step 5. The identification rules proposed in Eq. (25) and Eq. (26) can be used to identify the set of experts who need to change their preferences, denoted as $E S(Z)$, and the position $P\left(e^{a}\right)$ that should be changed for the expert $e^{a} \cdot R_{a}^{*(Z)}(a=1,2, \ldots, n)$ can be regarded as a decision aid and fed back to the corresponding expert. Let $R_{a}^{(Z+1)}=\left(r_{i j}^{a,(Z+1)}\right)_{m \times m}(a=1,2, \ldots, n)$ be the adjusted DHLPRs provided by experts. We can send the following suggestions to experts:

$$
r_{i j}^{a,(Z+1)} \in\left[\min \left\{r_{i j}^{a,(Z)}, r_{i j}^{{ }^{*} a,(Z)}\right\}, \max \left\{r_{i j}^{a,(Z)}, r_{i j}^{* a,(Z)}\right\}\right], \quad a=1,2, \ldots, n .
$$

Then, the experts are advised to change their preferences. Let $Z=Z+1$, and go back to Step 2. 


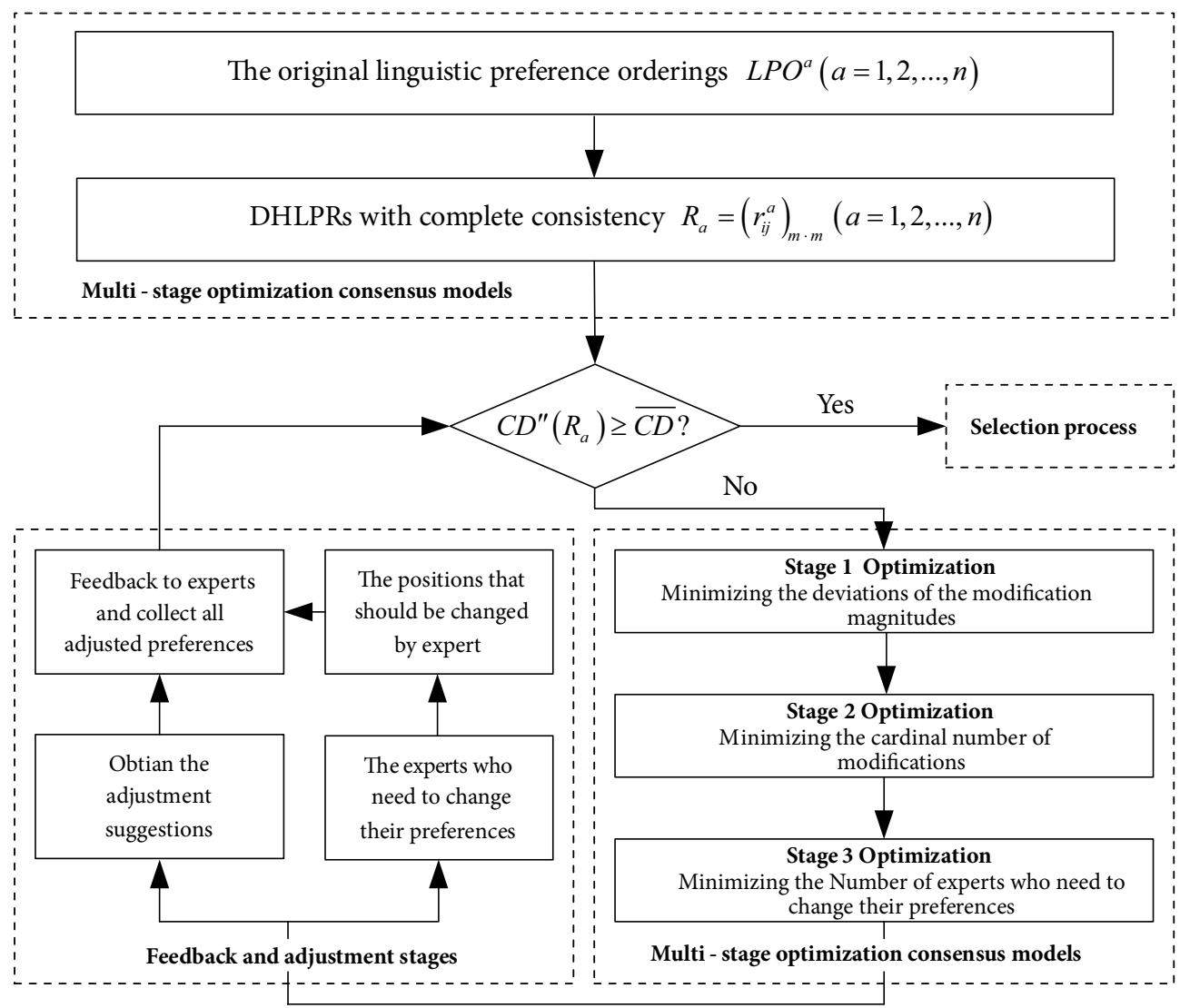

Figure 2. Multi-stage interactive group consensus optimization process with LPOs

Step 6. Let ${ }^{\star} R_{a}=R_{a}^{(Z)}(a=1,2, \ldots, n)$. Based on Definition 3.1, we obtain the collective DHLPR ${ }^{*} R_{c}=\left({ }^{*} r_{i j}^{c}\right)_{m \times m}$. Then, we calculate the synthetical value of each alternative $S V\left(A_{i}\right)=\sum_{j=1}^{m} f\left({ }^{\star}{ }_{i j}^{c}\right)$. Then we obtain the rank of all alternatives by ranking the synthetical values in descending order.

Step 7. End.

One Figure 2 is drawn to show the multi-stage interactive consensus reaching algorithm with LPOs.

\section{Numerical examples and comparative analyses}

This section mainly sets up two numerical examples to show the proposed transformation methods and the multi-stage interactive consensus reaching algorithm. Then, some comparative analyses with some existing methods are made to show the advantages and effectiveness of the proposed methods. 


\subsection{Numerical examples about transformation methods and comparative analyses}

Let $S_{O}=\left\{s_{t<o_{k}>} \mid t=-4, \ldots, 4 ; k=-4, \ldots, 4\right\}$. Suppose that two experts provide their LPOs for five alternatives $\left\{A_{1}, A_{2}, \ldots, A_{5}\right\}$ as follows:

$$
\begin{aligned}
& L P O^{1}=\left\{\begin{array}{cccc}
s_{0<o_{2}>} & s_{2<o_{1}>} & s_{1<o_{-1}>}>s_{0<o_{1}>} \\
A_{5}>A_{2}>A_{3}>A_{1}>A_{4}
\end{array}\right\},
\end{aligned}
$$

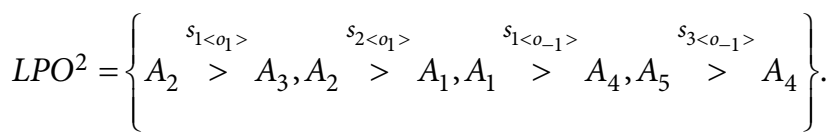

Firstly, based on Eq. (12) or (13), the LPOs can be transformed into the corresponding DHLPRs $R^{1}=\left(r_{i j}^{1}\right)_{5 \times 5}$ and $R^{2}=\left(r_{i j}^{2}\right)_{5 \times 5}$, respectively. Then, based on the transformation function $f$, two DHLPRs are transformed into the corresponding FPRs $P^{1}=\left(p_{i j}^{1}\right)_{5 \times 5}$ and $P^{2}=\left(p_{i j}^{2}\right)_{5 \times 5}$, where $p_{i j}^{k}+p_{j i}^{k}=1$ and $p_{i i}^{k}=0.5(i, j=1,2, \ldots, 5 ; k=1,2)$. Based on the transformation function $f$ and other transformation functions $g^{k}(k=1,2,3,4)$ (Chiclana et al., 1998; Dombi, 1995), we can transform $L P O^{1}$ into four corresponding FPRs $P^{1 k}=\left(p_{i j}^{1 k}\right)_{5 \times 5}$ $(k=1,2,3,4)$. The transformation results are shown in Table 2.

Based on Table 2, some comparative analyses are summarized as follows:

(1) As we know, the DHLPRs transformed from LPOs are completely consistent. Additionally, in this transformation process, we do not lose any original linguistic information. Furthermore, based on the transformation function $f$, two DHLPRs are transformed into the corresponding FPRs with complete consistencies.

(2) For the transformation function $g^{1}$, it is obvious that the FPR $P^{11}$ does not reflect the case when there is an indifference between two alternatives. Additionally, the transformed FPR only uses the elements 0 and 1 to represent the relationship between two alternatives, but it cannot reflect the degree (the DHLT) to which one alternative is superior to another. Finally, the transformation function $g^{1}$ cannot ensure that the transformed FPR $P^{11}$ is with complete consistency.

(3) Even though the transformation function $g^{2}$ can reflect the case when there is an indifference between two alternatives, both it cannot reflect any kind of intensity of preference between alternatives when we compare the pair of alternatives, and cannot ensure that the transformed FPR $P^{12}$ is with complete consistency.

(4) It is clear that both the transformation functions $g^{3}$ and $g^{4}$ are equal. Both of them use the utility values to reflect the degree (the DHLT) to which one alternative is superior to another, but the degree has a certain proportion and cannot reflect the real relationship of any two alternatives. Additionally, it also cannot ensure that the transformed FPRs $P^{13}$ and $P^{14}$ are with complete consistency.

In conclusion, the transformation functions $g^{k}(k=1,2,3,4)$ have different shortcomings. The transformation function proposed in this paper can not only ensure that the DHLPRs and FPRs are with complete consistencies, but also fully keep the original linguistic information (DHLTSs) unchanged. 
Table 2. The transformation results based on different methods

\begin{tabular}{|c|c|c|}
\hline Methods & Transformation results based on $L P O^{1}$ & Transformation results based on $L P O^{2}$ \\
\hline 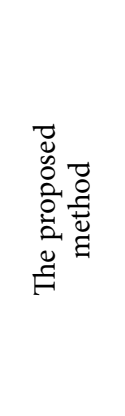 & 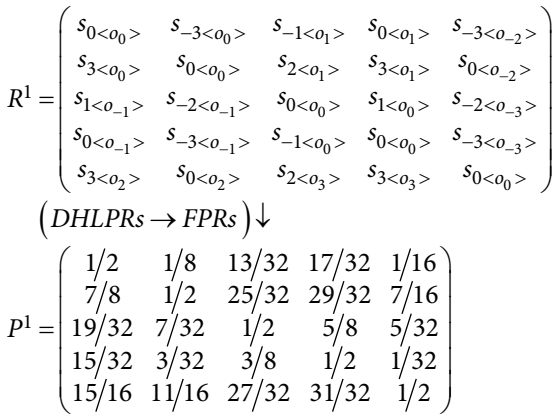 & 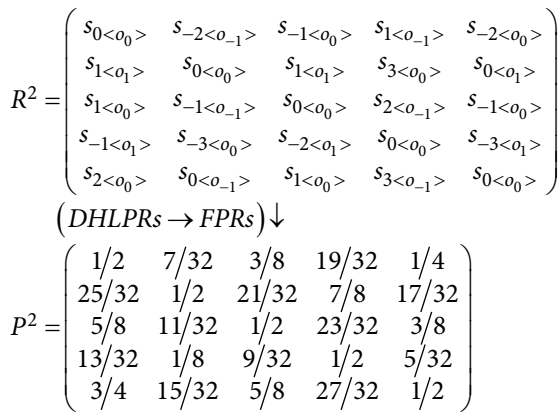 \\
\hline 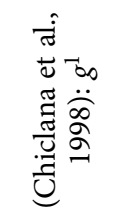 & $P^{11}=\left(\begin{array}{ccccc}- & 0 & 0 & 1 & 0 \\
1 & - & 1 & 1 & 0 \\
1 & 0 & - & 1 & 0 \\
0 & 0 & 0 & - & 0 \\
1 & 1 & 1 & 1 & -\end{array}\right)$ & - \\
\hline 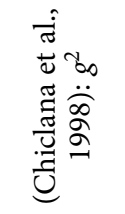 & $P^{12}=\left(\begin{array}{ccccc}1 / 2 & 0 & 0 & 1 & 0 \\
1 & 1 / 2 & 1 & 1 & 0 \\
1 & 0 & 1 / 2 & 1 & 0 \\
0 & 0 & 0 & 1 / 2 & 0 \\
1 & 1 & 1 & 1 & 1 / 2\end{array}\right)$ & - \\
\hline 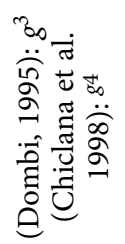 & $P^{13}=P^{14}=\left(\begin{array}{ccccc}1 / 2 & 3 / 4 & 5 / 8 & 3 / 8 & 7 / 8 \\
1 / 4 & 1 / 2 & 3 / 8 & 1 / 8 & 5 / 8 \\
3 / 8 & 5 / 8 & 1 / 2 & 1 / 4 & 3 / 4 \\
5 / 8 & 7 / 8 & 3 / 4 & 1 / 2 & 1 \\
1 / 8 & 3 / 8 & 1 / 4 & 0 & 1 / 2\end{array}\right)$ & - \\
\hline
\end{tabular}

\subsection{Numerical examples about consensus model and comparative analyses}

Suppose that a logistics company needs to choose the most suitable supplier from five competitive alternatives $\left\{A_{1}, A_{2}, \ldots, A_{5}\right\}$. To do so, four experts $\left\{e^{1}, e^{2}, e^{3}, e^{4}\right\}$ are invited to evaluate these five suppliers. These four experts provide their preferences with LPOs $\operatorname{LPO}^{a}(a=1,2,3,4)$ based on DHLTS $S_{O}=\left\{s_{t<o_{k}>} \mid t=-4, \ldots, 4 ; k=-4, \ldots, 4\right\}$ with $S=\left\{s_{-4}=\right.$ extremely bad, $s_{-3}=$ very bad,$s_{-2}=$ bad, $s_{-1}=$ slightly bad, $s_{0}=$ medium,

$s_{1}=$ slightly good, $s_{2}=$ good, $s_{3}=$ very good, $s_{4}=$ extremely good $\}$ and $O=\left\{o_{-4}=\right.$ far from,

$o_{-3}=$ scarcely, $o_{-2}=$ only a little, $o_{-1}=$ a little, $o_{0}=$ just right,$o_{1}=$ much, $o_{2}=$ very much,

$o_{3}=$ extremely much, $o_{4}=$ entirely $\}$.

$$
L P O^{1}=\left\{\begin{array}{cccc}
s_{0<o_{2}>} & s_{1<o_{1}>} & s_{1<o_{-1}>}> & s_{0<o_{1}>} \\
A_{5} & > & A_{2} & >
\end{array} A_{3}>A_{1}>A_{4}\right\},
$$




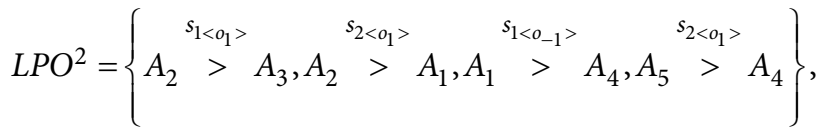

$$
\begin{aligned}
& L P O^{3}=\left\{\begin{array}{cccc}
s_{0<o_{1}>} & s_{2<o_{0}>} & s_{1<o_{-2}>} & s_{0<0_{3}>} \\
A_{3}>A_{5} & > & A_{2} & >
\end{array} A_{4}>A_{1}\right\}
\end{aligned}
$$

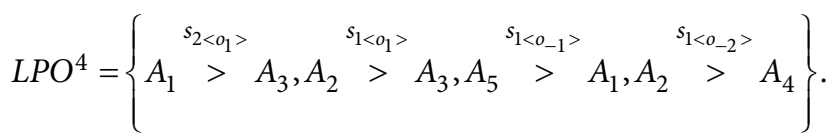

Obviously, this is an actual MEDM problem. Based on Table 1, we have $\overline{C I}=0.8256$, and according to Remark 4.1, we can let $\overline{C D}=0.85$ and $Z_{\max }=5$. But in the multi-stage consensus optimization model, to obtain the efficient intervals and send experts more helpful suggestions, we set $\overline{C I}=0.9$ and $\overline{C D}=0.9$.

Using Algorithm 4.1, the consensus reaching process is shown as follows:

Step 1. Transform all LPOs $L P O^{a}(a=1,2,3,4)$ into the DHLPRs $R_{a}=\left(r_{i j}^{a}\right)_{5 \times 5}(a=1,2,3,4)$ :

$$
\begin{aligned}
& R_{1}=\left(\begin{array}{ccccc}
s_{0<o_{0}>} & s_{-2<o_{0}>} & s_{-1<o_{1}>} & s_{0<o_{1}>} & s_{-2<o_{-2}}> \\
s_{2<o_{0}>} & s_{0<o_{0}>} & s_{1<o_{1}>} & s_{2<o_{1}>} & s_{0<o_{-2}>} \\
s_{1<o_{-1}>}> & s_{-1<o_{-1}>} & s_{0<o_{0}>} & s_{1<o_{0}>} & s_{-1<o_{-3}>} \\
s_{0<o_{-1}>}> & s_{-2<o_{-1}>}> & s_{-1<o_{0}>} & s_{0<o_{0}>} & s_{-2<o_{-3}>} \\
s_{2<o_{2}>} & s_{0<o_{2}>} & s_{1<o_{3}>} & s_{2<o_{3}>} & s_{0<o_{0}>}
\end{array}\right), \\
& R_{2}=\left(\begin{array}{ccccc}
s_{0<o_{0}>} & s_{-2<o_{-1}>} & s_{-1<o_{0}>} & s_{1<o_{-1}>} & s_{-1<o_{-2}>}> \\
s_{2<o_{1}>} & s_{0<o_{0}>} & s_{1<o_{1}>} & s_{3<o_{0}>} & s_{1<o_{-1}>} \\
s_{1<o_{0}>} & s_{-1<o_{-1}>} & s_{0<o_{0}>} & s_{2<o_{-1}>} & s_{0<o_{-2}>} \\
s_{-1<o_{1}>} & s_{-3<o_{0}>} & s_{-2<o_{1}>} & s_{0<o_{0}>} & s_{-2<o_{-1}>} \\
s_{1<o_{2}>} & s_{-1<o_{1}>} & s_{0<o_{2}>} & s_{2<o_{1}>} & s_{0<o_{0}>}
\end{array}\right), \\
& R_{3}=\left(\begin{array}{ccccc}
s_{0<o_{0}>} & s_{-1<o_{-1}>} & s_{-3<o_{-2}>} & s_{0<o_{-3}>} & s_{-3<o_{-1}>} \\
s_{1<o_{1}>} & s_{0<o_{0}>} & s_{-2<o_{-1}>} & s_{1<o_{-2}>} & s_{-2<o_{0}>} \\
s_{3<o_{2}>} & s_{2<o_{1}>} & s_{0<o_{0}>} & s_{3<o_{-1}>} & s_{0<o_{1}>} \\
s_{0<o_{3}>} & s_{-1<o_{2}>} & s_{-3<o_{1}>} & s_{0<o_{0}>} & s_{-3<o_{2}>} \\
s_{3<o_{1}>} & s_{2<o_{0}>} & s_{0<o_{-1}>} & s_{3<o_{-2}>} & s_{0<o_{0}>}
\end{array}\right), \\
& R_{4}=\left(\begin{array}{ccccc}
s_{0<o_{0}>} & s_{1<o_{0}>} & s_{2<o_{1}>} & s_{2<o_{-2}>} & s_{-1<o_{1}>} \\
s_{-1<o_{0}>} & s_{0<o_{0}>} & s_{1<o_{1}>} & s_{1<o_{-2}>} & s_{-2<o_{1}>} \\
s_{-2<o_{-1}>} & s_{-1<o_{-1}>} & s_{0<o_{0}>} & s_{0<o_{-3}>} & s_{-3<o_{0}>} \\
s_{-2<o_{2}>} & s_{-1<o_{2}>} & s_{0<o_{3}>} & s_{0<o_{0}>} & s_{-1<o_{1}>} \\
s_{1<o_{-1}>} & s_{2<o_{-1}>} & s_{3<o_{0}>} & s_{1<o_{-1}>} & s_{0<o_{0}>}
\end{array}\right) .
\end{aligned}
$$

Step 2. Let $Z=1$, and $R_{a}^{(1)}=\left(r_{i j}^{a,(1)}\right)_{5 \times 5}=\left(r_{i j}^{a}\right)_{5 \times 5}(a=1,2,3,4)$.

Step 3. Based on Eq. (17), we can obtain the consensus degree $C D^{\prime \prime}\left(R_{a}^{(1)}\right)$ for each DHLPR 
$R_{a}^{(1)}$, which is shown in Table 3. Additionally, considering that all DHLPRs are completely consistent, so the consistency indices of them are $C I\left(R_{a}^{(1)}\right)=1(a=1,2,3,4)$.

Table 3. The consistency indices and consensus degrees of DHLPRs $R_{a}^{(1)}(a=1,2,3,4)$

\begin{tabular}{|c|c|c|c|c|}
\hline & $R_{1}^{(1)}$ & $R_{2}^{(1)}$ & $R_{3}^{(1)}$ & $R_{4}^{(1)}$ \\
\hline$C I\left(R_{a}^{(1)}\right)$ & 1 & 1 & 1 & 1 \\
\hline$C D^{\prime \prime}\left(R_{a}^{(1)}\right)$ & 0.8354 & 0.8188 & 0.7563 & 0.7438 \\
\hline$C D^{\prime \prime}$ & \multicolumn{3}{|c|}{0.7438} \\
\hline
\end{tabular}

Obviously, $C D^{\prime \prime}=0.7438<0.85$ and the consensus is not reached. Therefore, we utilize the multi-stage consensus optimization model to improve the group consensus degree. In this paper, the optimization model is in continuous scale, that is $r_{i j}^{* a}{ }^{*}(Z) \in \bar{S}_{O}=\left\{s_{t<o_{k}>} \mid t=[-4,4]\right.$; $k=[-4,4]\}(a=1,2,3,4 ; i, j=1,2, \ldots, 5)$.

\section{Interactive consensus reaching process: Round 1}

Step $4^{\mathbf{1}}$. Let $G_{q}^{(Z)}, N_{q}^{(Z)}, N E_{q}^{(Z)}$ be the size of change, the modification number of the DHLTs in all DHLPRs, and the number of experts that have to change their preferences respectively at the stages $q(q=1,2,3)$ in the round $Z$. Using Eqs (22), (24), and (26), we have

$$
\begin{aligned}
& G_{1}^{(1)}=3.0938, N_{1}^{(1)}=21, N E_{1}^{(1)}=4 ; \\
& G_{2}^{(1)}=3.0938, N_{2}^{(1)}=20, N E_{2}^{(1)}=3 ; \\
& G_{3}^{(1)}=3.0938, N_{3}^{(1)}=20, N E_{3}^{(1)}=3 .
\end{aligned}
$$

Because $N E_{2}^{(1)}=N E_{3}^{(1)}=3$, there exists no any further improvement in this improvement process. Then $R_{1}^{*(1)}=R_{1}$ and the optimal adjusted DHLPRs can be shown as follows:

$$
\begin{aligned}
& R_{2}^{*}(1)=\left(\begin{array}{ccccc}
s_{0<o_{0}>} & s_{-2<o_{-1}>} & s_{-1<o_{0}>} & s_{1<o_{-1}>} & s_{-1<o_{-2}>} \\
s_{2<o_{1}>} & s_{0<o_{0}>} & s_{0<o_{-1.05}>}> & \frac{s_{0<o_{-2.47}>}>}{s_{0<o_{-2.98}>}} & \frac{s_{1<o_{0}>}}{s_{0<o_{-3.51}>}} \\
s_{0<o_{1.05}>} & s_{0<o_{0}>} & s_{2<o_{-1}>} & \frac{s_{0<o_{1}}}{s_{-2<o_{-1}>}} \\
s_{-1<o_{1}>}> & s_{0<o_{2.47}>}> & s_{-2<o_{1}>} & s_{0<o_{0}>} & s_{0<o_{0}>}
\end{array}\right),
\end{aligned}
$$

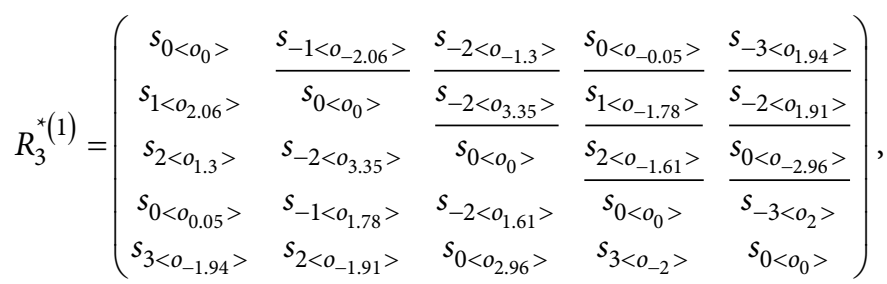




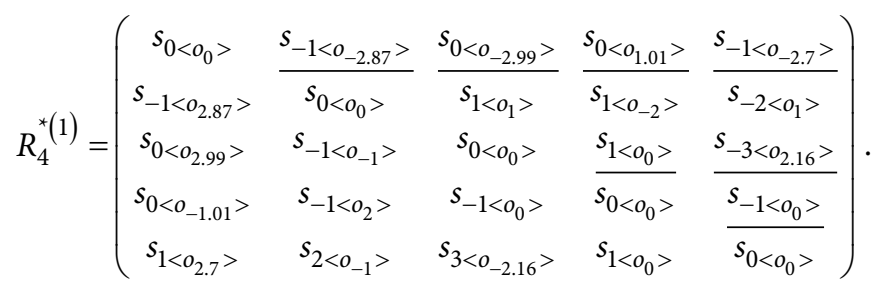

The consistency indices and consensus degrees of all the optimal adjusted DHLPRs $R_{a}^{*(1)}(a=1,2,3,4)$ in this stage are shown in Table 4 .

Table 4. The consistency indices and consensus degrees of all the optimal adjusted DHLPRs $R_{a}^{*(1)}(a=1,2,3,4)$

\begin{tabular}{|c|c|c|c|c|}
\hline & $R_{1}^{*(1)}$ & $R_{2}^{*(1)}$ & $R_{3}^{*(1)}$ & $R_{4}^{*(1)}$ \\
\hline$C I\left(R_{a}^{*(1)}\right)$ & 1 & 0.9 & 0.9689 & 0.9 \\
\hline$C D^{\prime \prime}\left(R_{a}^{*}(1)\right)$ & 0.9 & 0.9 & 0.9 & 0.9 \\
\hline$C D^{\prime \prime}$ & \multicolumn{2}{|c}{0.9} \\
\hline
\end{tabular}

Then, the optimal adjusted DHLPRs $R_{a}^{*(1)}(a=1,2,3,4)$ can be fed back to experts for improving the group consensus degrees.

Step $\mathbf{5}^{\mathbf{1}}$. With the help of the suggestions obtained above, the experts can decide how to adjust their preferences.

Based on the results obtained in the multi-stage optimization model, the experts $e^{2}, e^{3}$, and $e^{4}$ need to improve their preferences. Combining $R_{a}^{(1)}(a=2,3,4)$ and $R_{a}^{*(1)}(a=2,3,4)$, the suggestions can be listed as follows:

For the expert $e^{2}: r_{23}^{2,(2)} \in\left[S_{0<o_{-1.05}>}, S_{1<o_{1}>}\right], r_{24}^{2,(2)} \in\left[S_{0<o_{-2.47}>}, S_{3<o_{0}>}\right]$,

$r_{25}^{2,(2)} \in\left[S_{0<o_{-2.98}>}, S_{1<o_{-1}>}\right]$, and $r_{35}^{2,(2)} \in\left[S_{0<o_{-3.51}>}, S_{0<o_{-2}>}\right]$.

For the expert $e^{3}: r_{12}^{3,(2)} \in\left[s_{-1<o_{-2.06}>}, s_{-1<o_{-1}>}\right], r_{13}^{3,(2)} \in\left[s_{-3<o_{-2}>}, s_{-2<o_{-1.3}>}\right]$,

$r_{14}^{3,(2)} \in\left[s_{0<o_{-3}>}, s_{0<o_{-0.05}>}\right], r_{15}^{3,(2)} \in\left[s_{-3<o_{-1}>}, s_{-3<o_{1.94}>}\right], r_{23}^{3,(2)} \in\left[s_{-2<o_{-1}>}, s_{-2<o_{3.35}>}\right]$,

$r_{24}^{3,(2)} \in\left[s_{1<o_{-2}>}, S_{1<o_{-1.78}>}\right], r_{25}^{3,(2)} \in\left[s_{-2<o_{0}>}, S_{-2<o_{1.91}>}\right]$, and

$r_{34}^{3,(2)} \in\left[s_{2<o_{-1.61}>}, S_{3<o_{-1}>}\right], r_{35}^{3,(2)} \in\left[S_{0<o_{-2.96}>}, S_{0<o_{1}>}\right]$.

For the expert $e^{4}: r_{12}^{4,(2)} \in\left[S_{-1<o_{-2.87}>}, S_{1<o_{0}>}\right], r_{13}^{4,(2)} \in\left[S_{0<o_{-2.99}>}, S_{2<o_{1}>}\right]$,

$r_{14}^{4,(2)} \in\left[S_{0<o_{1.01}>}, s_{2<o_{-2}>}\right], r_{15}^{4,(2)} \in\left[S_{-1<o_{-2.7}>}, S_{-1<o_{1}>}\right], r_{34}^{4,(2)} \in\left[s_{0<o_{-3}>}, S_{1<o_{0}>}\right]$,

$r_{35}^{4,(2)} \in\left[S_{-3<o_{0}>}, S_{-3<0_{2.16}>}\right]$, and $r_{45}^{4,(2)} \in\left[S_{-1<o_{0}>}, S_{-1<o_{1}>}\right]$. 
Suppose that the experts provide their adjusted preferences as follows:

For the expert $e^{2}: r_{23}^{2,(2)}=s_{0<o_{-2}>}, r_{24}^{2,(2)}=s_{0<o_{0}>}, r_{25}^{2,(2)}=s_{1<o_{-2}>}$, and $r_{35}^{2,(2)}=s_{0<o_{-3}>}$.

For the expert $e^{3}: r_{12}^{3,(2)}=s_{-1<o_{-2}>}, r_{13}^{3,(2)}=s_{-3<o_{1}>}, r_{14}^{3,(2)}=s_{0<o_{-1}>}, r_{15}^{3,(2)}=s_{-3<o_{1}>}$,

$$
r_{23}^{3,(2)}=s_{-2<o_{2}>}, r_{25}^{3,(2)}=s_{-2<o_{1}>} \text {, and } r_{34}^{3,(2)}=s_{2<o_{1}>}, r_{35}^{3,(2)}=s_{0<o_{-1}>} \text {. }
$$

For the expert $e^{4}: r_{12}^{4,(2)}=s_{-1<o_{1}>}, r_{13}^{4,(2)}=s_{0<o_{1}>}, r_{14}^{4,(2)}=s_{0<o_{3}>}, r_{15}^{4,(2)}=s_{-1<o_{-1}>}$,

$$
r_{34}^{4,(2)}=s_{0<0_{3}>} \text {, and } r_{35}^{4,(2)}=s_{-3<o_{1}>} \text {. }
$$

\section{Round 2}

Step $4^{2}$. The consistency indices and consensus degrees of the adjusted DHLPRs $R_{a}^{(2)}(a=1,2,3,4)$ can be obtained as follows (Table 5):

Table 5. The consensus degrees of the adjusted DHLPRs $R_{a}^{(2)}(a=1,2,3,4)$

\begin{tabular}{|c|c|c|c|c|}
\hline & $R_{1}^{(2)}$ & $R_{2}^{(2)}$ & $R_{3}^{(2)}$ & $R_{4}^{(2)}$ \\
\hline$C I\left(R_{a}^{(2)}\right)$ & 0.9539 & 0.9609 & 0.9654 & 0.9643 \\
\hline$C D^{\prime \prime}\left(R_{a}^{(2)}\right)$ & 0.8604 & 0.8625 & 0.8396 & 0.8208 \\
\hline$C D^{\prime \prime}$ & \multicolumn{2}{|c|}{0.8208} \\
\hline
\end{tabular}

Obviously, $C D^{\prime \prime}=0.8208<0.85$ and the consensus is also not reached. Therefore, we still need to utilize the multi-stage consensus optimization model to improve the group consensus degree. Then, we have

$$
\begin{aligned}
& G_{1}^{(2)}=1.1063, N_{1}^{(2)}=13, N E_{1}^{(2)}=4 ; \\
& G_{2}^{(2)}=1.1063, N_{2}^{(2)}=11, N E_{2}^{(2)}=4 ; \\
& G_{3}^{(2)}=1.1063, N_{3}^{(2)}=11, N E_{3}^{(2)}=4 .
\end{aligned}
$$

The optimal adjusted DHLPRs are shown as follows:

$$
\begin{aligned}
R_{1}^{(2)} & =\left(\begin{array}{ccccc}
s_{0<o_{0}>} & s_{-2<o_{0}>} & s_{-1<o_{1}>} & s_{0<o_{1}>} & s_{-2<o_{-2}>} \\
s_{2<o_{0}>} & s_{0<o_{0}>} & s_{1<o_{1}>} & s_{2<o_{-0.3}>} & s_{0<o_{-2}>} \\
s_{1<o_{-1}>} & s_{-1<o_{-1}>} & s_{0<o_{0}>} & s_{1<o_{0}>} & s_{-1<o_{-3}>} \\
s_{0<o_{-1}>} & s_{-2<o_{-1}>}> & s_{-1<o_{0}>} & s_{0<o_{0}>} & s_{-2<o_{-3}>} \\
s_{2<o_{2}>} & s_{0<o_{2}>} & s_{1<o_{3}>} & s_{2<o_{3}>} & s_{0<o_{0}>}
\end{array}\right), \\
R_{2}^{*(2)}= & \left(\begin{array}{ccccc}
s_{0<o_{0}>}> & s_{-2<o_{-1}>}> & s_{-1<o_{0}>}> & s_{1<o_{-1}>} & s_{-1<o_{-2}>}> \\
s_{2<o_{1}>} & s_{0<o_{0}>} & s_{0<o_{-2}>}> & s_{0<o_{0}>} & s_{0<o_{1.7}>} \\
s_{1<o_{0}>} & s_{0<o_{2}>} & s_{0<o_{0}>} & s_{2<o_{-1}>} & \frac{s_{-1<o_{-0.1}>}}{s_{-2<o_{-1}>}>} \\
s_{-1<o_{1}>} & s_{0<o_{0}>} & s_{-2<o_{1}>}> & s_{0<o_{0}>} & s_{0<o_{0}>}
\end{array}\right),
\end{aligned}
$$




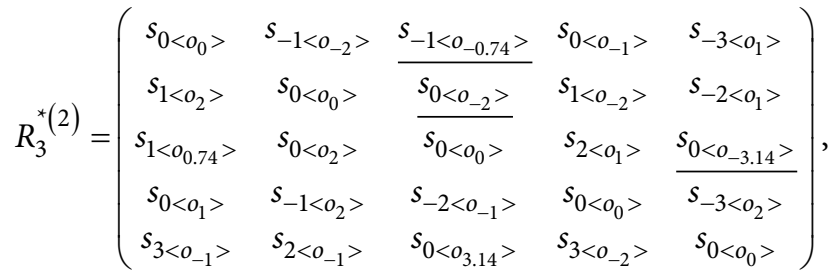

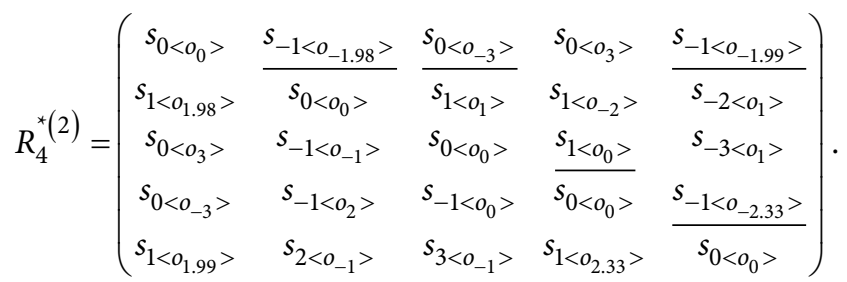

The consistency indices and consensus degrees of all the optimal adjusted DHLPRs $R_{a}^{*(2)}(a=1,2,3,4)$ in this stage are obtained as follows (Table 6):

Table 6. The consistency levels and consensus levels of all the optimal adjusted DHLPRs $R_{a}^{*}(2)(a=1,2,3,4)$

\begin{tabular}{|c|c|c|c|c|}
\hline & $R_{1}^{*(2)}$ & $R_{2}^{*(2)}$ & $R_{3}^{*(2)}$ & $R_{4}^{*(2)}$ \\
\hline$C I\left(R_{a}^{*(2)}\right)$ & 0.9919 & 0.9 & 0.9445 & 0.9 \\
\hline$C D^{\prime \prime}\left(R_{a}^{*(2)}\right)$ & 0.9 & 0.9 & 0.9 & 0.9 \\
\hline$C D^{\prime \prime}$ & \multicolumn{2}{|c|}{0.9} \\
\hline
\end{tabular}

Step $5^{2}$. Based on the results obtained in multi-stage optimization model in this round, all the experts need to adjust their preferences, and the suggestions are:

For the expert $e^{1}: r_{24}^{1,(3)} \in\left[s_{2<o_{-0.3}>}, S_{2<o_{1}>}\right]$.

For the expert $e^{2}: r_{25}^{2,(3)} \in\left[s_{0<o_{1.7}>}, S_{1<o_{-2}>}\right]$, and $r_{35}^{2,(3)} \in\left[S_{-1<o_{-0.1}>}, S_{0<o_{-3}>}\right]$.

For the expert $e^{3}: r_{13}^{3,(3)} \in\left[S_{-3<o_{1}>}, S_{-1<o_{-0.74}>}\right], r_{23}^{3,(4)} \in\left[S_{-2<o_{2}>}, S_{0<o_{-2}>}\right]$, and $r_{35}^{3,(3)} \in\left[S_{0<o_{-3.14}>}, S_{0<o_{-1}>}\right]$.

For the expert $e^{4}: r_{12}^{4,(3)} \in\left[S_{-1<o_{-1.98}>}, S_{1<o_{1}>}\right], r_{13}^{4,(3)} \in\left[S_{0<o_{-3}>}, S_{0<o_{1}>}\right]$, $r_{15}^{4,(3)} \in\left[S_{-1<o_{-1.99}>}, S_{-1<o_{-1}>}\right]$, and $r_{34}^{4,(3)} \in\left[S_{0<o_{3}>}, S_{1<o_{0}>}\right]$, $r_{45}^{4,(3)} \in\left[S_{-1<o_{-2.33}>}, S_{-1<o_{-1}>}\right]$.

Suppose that the experts provide their adjusted preferences as follows:

For the expert $e^{1}: r_{24}^{1,(3)}=S_{2<o_{0}>}$

For the expert $e^{2}: r_{35}^{2,(3)}=S_{-1<o_{0}>}$. 
For the expert $e^{3}: r_{13}^{3,(3)}=s_{-1<o_{-2}>}, r_{23}^{3,(3)}=s_{-1<o_{0}>}$, and $r_{35}^{3,(3)}=s_{0<o_{-3}>}$.

For the expert $e^{4}: r_{12}^{4,(3)}=s_{-1<o_{2}>}, r_{13}^{4,(3)}=s_{0<o_{-1}>}, r_{34}^{4,(3)}=s_{1<o_{0}>}$, and $r_{45}^{4,(3)}=s_{-1<o_{-2}>}$.

\section{Round 3}

The consensus degrees of the adjusted DHLPRs can be obtained as:

Table 7. The group consensus index of the adjusted DHLPRs $R_{a}^{(3)}(a=1,2,3,4)$

\begin{tabular}{|c|c|c|c|c|}
\hline & $R_{1}^{(3)}$ & $R_{2}^{(3)}$ & $R_{3}^{(3)}$ & $R_{4}^{(3)}$ \\
\hline$C I\left(R_{a}^{(3)}\right)$ & 0.9552 & 0.9617 & 0.9630 & 0.9659 \\
\hline$C D^{\prime \prime}\left(R_{a}^{(3)}\right)$ & 0.8875 & 0.8875 & 0.8813 & 0.8729 \\
\hline$C D^{\prime \prime}$ & \multicolumn{2}{|c|}{0.8729} \\
\hline
\end{tabular}

In Table 7, there is $C D^{\prime \prime}=0.8729>0.85$, so all experts have reached the group consensus and the interactive consensus reaching process is terminated. Additionally, the consistency indices of all DHLPRs also satisfy the given threshold value.

Step 6. Based on Definition 3.1, we obtain the collective DHLPR and calculate the synthetical value of each alternative $S V\left(A_{1}\right)=1.9922, S V\left(A_{2}\right)=2.7109, S V\left(A_{3}\right)=2.5703$, $S V\left(A_{4}\right)=1.8906, S V\left(A_{5}\right)=3.3359$. Then we can obtain the ranking of all alternatives: $A_{5}>A_{2}>A_{3}>A_{1}>A_{4}$.

Step 7. End.

Next, we can utilize the existing methods to deal with this MEDM problem:

(1) We can use the method introduced by $\mathrm{He}$ and $\mathrm{Xu}$ (2018) to deal with this GDM problem. Firstly, we need to transform $L P O^{1}$ and $L P O^{3}$ into the corresponding POs by deleting the DHLTs. Additionally, it is difficult to obtain the corresponding POs of $L P O^{2}$ and $L P O^{4}$ directly, but we can get them by the transformed DHLPRs. The final POs of these four LPOs are shown as follows:

$$
P O^{1}=\{4,2,3,5,1\}, P O^{2}=\{4,1,3,5,2\}, P O^{3}=\{5,3,1,4,2\}, P O^{4}=\{2,3,5,4,1\} .
$$

Based on the consensus measure proposed by $\mathrm{He}$ and $\mathrm{Xu}$ (2018), we can obtain the decision-making result as $A_{5}>A_{2}>A_{3}>A_{1}>A_{4}$ based on both the section process and consensus process.

(2) We can also utilize the method proposed by Chiclana et al. (1998) to deal with this MEDM problem. Firstly, based on the transformation function $f$, four LPOs can be transformed to FPR $P^{k}(k=1,2,3,4)$. Then, we can obtain the collective FPR $P^{c}=\left(p_{i j}^{c}\right)_{5 \times 5}$, and compute the quantifier-guided dominance degree $Q G D D_{i}$ and the quantifier guided non-dominance degree $Q G N D D_{i}$ of each alternative $A_{i}$. The decision-making result is shown in Table 8. 
Table 8. The $Q G D D_{i}$ and $Q G N D D_{i}$ of all alternatives $A_{i}(i=1,2, \ldots, 5)$

\begin{tabular}{|c|c|c|c|c|c|c|}
\hline & $A_{1}$ & $A_{2}$ & $A_{3}$ & $A_{4}$ & $A_{5}$ & Rank \\
\hline$Q_{G D D}$ & 0.3926 & 0.5684 & 0.5098 & 0.3359 & 0.6934 & $A_{5}>A_{2}>A_{3}>A_{1}>A_{4}$ \\
\hline$Q G N D D_{i}$ & 0.7578 & 0.9453 & 0.8984 & 0.6719 & 1 & $A_{5}>A_{2}>A_{3}>A_{1}>A_{4}$ \\
\hline Final rank & \multicolumn{6}{|c|}{$A_{5}>A_{2}>A_{3}>A_{1}>A_{4}$} \\
\hline
\end{tabular}

Based on multi-stage consensus optimization model and the existing methods ( $\mathrm{He} \& \mathrm{Xu}$, 2018; Chiclana et al., 1998), some comparative analyses are summarized as follows:

(a) He and $\mathrm{Xu}$ (2018) discussed a consensus framework with three kinds of preference orderings including Preference orderings (Chiclana et al., 1998), Interval preference orderings (IPOs) (González-Pachón \& Romero, 2001), and Hesitant preference orderings set (HPOS) (He \& Xu, 2018); Chiclana et al. (1998) discussed some transformation methods to transform preference orderings into FPRs. However, the firstly and most important gap of all the preference orderings proposed by $\mathrm{He}$ and $\mathrm{Xu}$ (2018) and Chiclana et al. (1998) do not exist any relationship between two adjacent alternatives, which leads to incomplete evaluation information. Additionally, $\mathrm{He}$ and $\mathrm{Xu}$ (2018) and Chiclana et al. (1998) only discussed some preference orderings in continuous forms, but ignored the preference orderings in decentralized forms. Furthermore, because all the preference orderings proposed by $\mathrm{He}$ and $\mathrm{Xu}$ (2018) and Chiclana et al. (1998) only consider the orderings among all alternatives and ignore the relationship between two adjacent alternatives, so the transformation methods given by Chiclana et al. (1998) only utilize the preference ordering to obtain the corresponding FPRs.

To overcome the gaps discussed above, we propose two kinds of LPOs. Firstly, the LPOs utilize the DHLTs to represent the relationships between two adjacent alternatives. Secondly, by fully considering the behaviors of the experts, we define two different LPOs in continuous forms and decentralized forms, respectively. Thirdly, combining the DHLTs and different forms of LPOs, the transformed DHLPRs will be more complete.

(b) In the transformation process, the transformation function proposed in this paper cannot only ensure that the DHLPRs and FPRs are with complete consistencies, but also fully consider the original linguistic information (DHLTSs). However, the transformation functions $g^{k}(k=1,2,3,4)$ discussed by Chiclana et al. (1998) cannot ensure that the transformed FPRs are with complete consistencies. Furthermore, even though some functions of them use the utility values to reflect the degree to which one alternative is superior to another, but the degree has a certain proportion and cannot reflect the real relationship of any two alternatives.

(c) In the consensus reaching process, firstly, we do not need to improve the consistencies of the transformed DHLPRs considering that all the transformed DHLPRs are completely consistent. Secondly, the multi-stage consensus optimization model proposed in this paper consists of three objectives: minimizing the deviations of the modification magnitudes, minimizing the cardinal number of modifications while keeping the value of the first objective constant, and minimizing the number of experts who need to change 
their evaluations. Therefore, this model can be used to achieve consensus using minimal changes in the size of the change, the number of modifications, and the number of individuals who need to revise their preferences.

The method proposed by $\mathrm{He}$ and $\mathrm{Xu}$ (2018) only discusses how to identify the expert with the smallest consensus degree and proposed an automatic adjustment method to improve the consensus degree. Therefore, the multi-stage consensus optimization model proposed in this paper is more efficient and targeted.

\section{Conclusions}

Linguistic preference orderings are the useful representation forms for reflecting the real thoughts of experts in MEDM problems. Additionally, transforming each LPO into the corresponding DHLPR with complete consistency equivalently is the preparation of the consensus reaching process. Finally, a multi-stage consensus optimization model is developed to provide more refined solutions to experts and help experts achieve consensus. The main contributions of this paper are as follows:

(1) We have defined two novel concepts of LPOs, which are in continuous form and in decentralized form, respectively.

(2) (We have developed two equivalent transformation models to transform LPOs into the corresponding DHLPRs with complete consistencies.

(3) We have established a multi-stage consensus optimization model by considering the suggested preferences represented in both the continuous scale and the discrete scale. Additionally, we have also developed a multi-stage interactive consensus reaching algorithm to deal with the MEDM problem with LPOs.

(4) We have made some comparative analyses between the proposed methods and models and some existing methods to show the advantages of the proposed methods and models.

However, this paper only researches the additive consistent DHLPR and discusses the general consensus reaching environment. As the future study, we will continue to investigate the other consistency methods for DHLPRs, such as multiplicative consistency and interval consistency, and use them to deal with LPOs. Additionally, we will also research some consensus models with LPOs under large-scale group decision making environment (Gou et al., 2019b; Song \& Hu, 2019; Song \& Li, 2019).

\section{Funding}

This study was supported by the National Natural Science Foundation of China under Grant [numbers 71571123, 71561026 and 71771155]; Major Program of the National Social Science Fund of China under Grant [number 17ZDA092]. 


\section{Author contributions}

Xunjie Gou conceived the study. Xunjie Gou wrote the first draft of the article. Wei Zhou was responsible for data collection and analysis. Zeshui Xu was responsible for supervision and funding acquisition.

\section{Disclosure statement}

The authors have no any competing financial, professional, or personal interests from other parties.

\section{References}

Beliakov, G., James, S. \& Wilkin, T. (2017). Aggregation and consensus for preference relations based on fuzzy partial orders. Fuzzy Optimization and Decision Making, 16(4), 409-428. https://doi.org/10.1007/s10700-016-9258-4

Ben-Arieh, D., \& Easton, T. (2007). Multi-criteria group consensus under linear cost opinion elasticity. Decision Support Systems, 43(3), 713-721. https://doi.org/10.1016/j.dss.2006.11.009

Chiclana, F., Herrera, F., \& Herrera-Viedma, E. (1998). Integrating three representation models in fuzzy multipurpose decision making based on fuzzy preference relations. Fuzzy Set and Systems, 97, 33-48. https://doi.org/10.1016/S0165-0114(96)00339-9

Del Moral, M. J., Chiclana, F., Tapia, J. M., \& Herrera-Viedma, E. (2018). A comparative study on consensus measures in group decision making. International Journal of Intelligent Systems, 33(8), 1624-1638. https://doi.org/10.1002/int.21954

Dombi, J. (1995). A general framework for the utility-based and outranking methods. In Fuzzy Logic and Soft Computing (pp. 202-208). World Scientific. https://doi.org/10.1142/9789812830753_0024

Dong, Y. C., Xu, Y. F., \& Li, H. Y. (2008). On consistency measures of linguistic preference relations. European Journal of Operational Research, 189(2), 430-444. https://doi.org/10.1016/j.ejor.2007.06.013

Fan, Z. P., Ma, J., Jiang, Y. P., Sun, Y. H., \& Ma, L. (2006). A goal programming approach to group decision making based on multiplicative preference relations and fuzzy preference relations. European Journal of Operational Research, 174(1), 311-321. https://doi.org/10.1016/j.ejor.2005.03.026

Fu, Z. G., \& Liao, H. C. (2019). Unbalanced double hierarchy linguistic term set: The TOPSIS method for multi-expert qualitative decision making involving green mine selection. Information Fusion, 51, 271-286. https://doi.org/10.1016/j.inffus.2019.04.002

González-Pachón, J., \& Romero, C. (2001). Aggregation of partial ordinal rankings: An interval goal programming approach. Computers \& Operations Research, 28, 827-834. https://doi.org/10.1016/S0305-0548(00)00010-1

Gou, X. J., \& Liao, H. C. (2019). About the double hierarchy linguistic term set and its extensions. ICSES Transactions on Neural and Fuzzy Computing, 2(2), 13-20.

Gou, X. J., Liao, H. C., Wang, X. X., Xu, Z. S., \& Herrera, F. (2020). Consensus based on multiplicative consistent double hierarchy linguistic preferences: Venture capital in real estate market. International Journal of Strategic Property Management, 24(01), 1-23. https://doi.org/10.3846/ijspm.2019.10431

Gou, X. J., Liao, H. C., Xu, Z. S., \& Herrera, F. (2017). Double hierarchy hesitant fuzzy linguistic term set and MULTIMOORA method: A case of study to evaluate the implementation status of haze controlling measures. Information Fusion, 38, 22-34. https://doi.org/10.1016/j.inffus.2017.02.008 
Gou, X. J., Liao, H. C., Xu, Z. S., \& Herrera, F. (2019a). Consensus model handling minority opinions and non-cooperative behaviors in large-scale group decision-making under double hierarchy linguistic preference relations (Technical report). IEEE Transactions on Cybernetics.

Gou, X. J., Liao, H. C., Xu, Z. S., Min, R., \& Herrera, F. (2019b). Group decision making with double hierarchy hesitant fuzzy linguistic preference relations: Consistency based measures, index and repairing algorithms and decision model. Information Sciences, 489, 93-112. https://doi.org/10.1016/j.ins.2019.03.037

Gou, X. J., Xu, Z. S., \& Herrera, F. (2018a). Consensus reaching process for large-scale group decision making with double hierarchy hesitant fuzzy linguistic preference relations. Knowledge-Based Systems, 157, 20-33. https://doi.org/10.1016/j.knosys.2018.05.008

Gou, X. J., Xu, Z. S., Liao, H. C., \& Herrera, F. (2018b). Multiple criteria decision making based on distance and similarity measures with double hierarchy hesitant fuzzy linguistic term sets. Computers \& Industrial Engineering, 126, 516-530. https://doi.org/10.1016/j.cie.2018.10.020

He, Y., \& Xu, Z. S. (2018). A consensus framework with different preference ordering structures and its applications in human resource selection. Computers \& Industrial Engineering, 118, 80-88. https://doi.org/10.1016/j.cie.2018.02.022

Herrera, F., \& Martínez, L. (2000). A 2-tuple fuzzy linguistic representation model for computing with words. IEEE Transactions on Fuzzy Systems, 8, 746-752. https://doi.org/10.1109/91.890332

Herrera-Viedma, E., Herrera, F., Chiclana, F., \& Luque, M. (2004). Some issues on consistency of fuzzy preference relations. European Journal of Operational Research, 154(1), 98-109. https://doi.org/10.1016/S0377-2217(02)00725-7

Herrera-Viedma, E., Martínez, L., Mata, F., \& Chiclana, F. (2005). A consensus support system model for group decision-making problems with multigranular linguistic preference relations. IEEE Transactions on Fuzzy Systems, 13(5), 644-658. https://doi.org/10.1109/TFUZZ.2005.856561

Hervés-Beloso, C., \& Cruces, H. V. (2018). Continuous preference orderings representable by utility functions. Journal of Economic Surveys, 33(1), 179-194. https://doi.org/10.1111/joes.12259

Kacprzyk, J., \& Fedrizzi, M. (1988). A 'soft' measure of consensus in the setting of partial (fuzzy) preferences. European Journal of Operational Research, 34(3), 316-325.

https://doi.org/10.1016/0377-2217(88)90152-X

Kamis, N. H., Chiclana, F., \& Levesley, J. (2018). Preference similarity network structural equivalence clustering based consensus group decision making model. Applied Soft Computing, 67, 706-720. https://doi.org/10.1016/j.asoc.2017.11.022

Kou, G., \& Lin, C. (2014). A cosine maximization method for the priority vector derivation in AHP. European Journal of Operational Research, 235(1), 225-232. https://doi.org/10.1016/j.ejor.2013.10.019

Krishankumar, R., Subrajaa, L. S., Ravichandran, K. S., Kar S., \& Saeid, A. B. (2019). A framework for multi-attribute group decision-making using double hierarchy hesitant fuzzy linguistic term set. International Journal of Fuzzy Systems, 21(4), 1130-1143. https://doi.org/10.1007/s40815-019-00618-w

Lan, J. B., Yang, M., Hu, M. M., \& Liu, F. (2018). Multi-attribute group decision making based on hesitant fuzzy sets, topsis method and fuzzy preference relations. Technological and Economic Development of Economy, 24(6), 2295-2317. https://doi.org/10.3846/tede.2018.6768

Liang, H. M., Xiong, W., \& Dong, Y. C. (2018). A prospect theory-based method for fusing the individual preference-approval structures in group decision making. Computers \& Industrial Engineering, 117, 237-248. https://doi.org/10.1016/j.cie.2018.01.001

Liao, H. C., Xu, Z. S., Zeng, X. J., \& Xu, D. L. (2016). An enhanced consensus reaching process in group decision making with intuitionistic fuzzy preference relations. Information Sciences, 329, 274-286. https://doi.org/10.1016/j.ins.2015.09.024 
Liu, N. N., He, Y., \& Xu, Z. S. (2019). Evaluate public-private-partnership's Advancement using double hierarchy hesitant fuzzy linguistic PROMETHEE with subjective and objective information from stakeholder perspective. Technological and Economic Development of Economy, 25(3), 386-420. https://doi.org/10.3846/tede.2019.7588

Meng, F. Y., Tang, J., \& Zhang, S. L. (2019). Interval linguistic fuzzy decision making in perspective of preference relations. Technological and Economic Development of Economy, 25(5), 998-1015. https://doi.org/10.3846/tede.2019.10548

Montserrat-Adell, J., Xu, Z. S., Gou, X. J., \& Agell, N. (2019). Free double hierarchy hesitant fuzzy linguistic term sets: An application on raking alternatives in GDM. Information Fusion, 47, 45-59. https://doi.org/10.1016/j.inffus.2018.07.002

Morente-Molinera, J. A., Kou, G., Pérez, I. J., Samuylov, K., Selamat, A., \& Herrera-Viedma, E. (2018). A group decision making support system for the Web: How to work in environments with a high number of participants and alternatives. Applied Soft Computing, 68, 191-201.

https://doi.org/10.1016/j.asoc.2018.03.047

Morente-Molinera, J. A., Kou, G., Samuylov, K., Ureña, R., \& Herrera-Viedma, E. (2019). Carrying out consensual group decision making processes under social networks using sentiment analysis over comparative expressions. Knowledge-Based Systems, 165, 335-345.

https://doi.org/10.1016/j.knosys.2018.12.006

Parreiras, R., Ekel, P., \& Bernardes, F. (2012). A dynamic consensus scheme based on a nonreciprocal fuzzy preference relation modeling. Information Sciences, 211, 1-17.

https://doi.org/10.1016/j.ins.2012.05.001

Schubert, J. (1995). On p in a decision-theoretic apparatus of Dempster-Shafer theory. International Journal of Approximate Reasoning, 13, 185-200. https://doi.org/10.1016/0888-613X(95)00061-K

Song, Y. M., \& Hu, J. (2019). Large-scale group decision making with multiple stakeholders based on probabilistic linguistic preference relation. Applied Soft and Computing, 80, 712-722. https://doi.org/10.1016/j.asoc.2019.04.036

Song, Y. M., \& Li, G. X. (2019). A large-scale group decision making with incomplete multi-granular probabilistic linguistic term sets and its application in sustainable supplier selection. Journal of the Operational Research Society, 70(5), 827-841. https://doi.org/10.1080/01605682.2018.1458017

Tanino, T. (1984). Fuzzy preference orderings in group decision making. Fuzzy Sets Systems, 12, 117131. https://doi.org/10.1016/0165-0114(84)90032-0

Tanino, T. (1988). Fuzzy preference relations in group decision making. In J. Kacprzyk \& M. Roubens (Eds.), Non-Conventional Preference Relations in Decision Making (pp. 54-71). Springer, Berlin. https://doi. org/10.1007/978-3-642-51711-2_4

Wan, S. P., Wang, F. \& Dong, J. Y. (2018). A group decision-making method considering both the group consensus and multiplicative consistency of interval-valued intuitionistic fuzzy preference relations. Information Sciences, 466, 109-128. https://doi.org/10.1016/j.ins.2018.07.031

Wu, Z. B., Huang, S., \& Xu, J. P. (2019a). Multi-stage optimization models for individual consistency and group consensus with preference relations. European Journal of Operational Research, 275, 182-194. https://doi.org/10.1016/j.ejor.2018.11.014

Wu, Z. B., Jin, B. M., \& Xu, J. P. (2018). Local feedback strategy for consensus building with probabilityhesitant fuzzy preference relations. Applied Soft Computing, 67, 691-705. https://doi.org/10.1016/j.asoc.2017.06.011

Wu, H. Y., Ren, P. J., \& Xu, Z. S. (2019b). Hesitant fuzzy linguistic consensus model based on trustrecommendation mechanism for hospital expert consultation. IEEE Transactions on Fuzzy Systems, 27(11), 2227-2241. https://doi.org/10.1109/TFUZZ.2019.2896836 
Wu, Z. B., \& Xu, J. P. (2018). A consensus model for large-scale group decision making with hesitant fuzzy information and changeable clusters. Information Fusion, 41, 217-231. https://doi.org/10.1016/j.inffus.2017.09.011

Xu, Y. J., Wen, X. W., \& Zhang, W. C. (2018). A two-stage consensus method for large-scale multiattribute group decision making with an application to earthquake shelter selection. Computers \& Industrial Engineering, 116, 113-129. https://doi.org/10.1016/j.cie.2017.11.025

Xu, Y. J., Herrera, F., \& Wang, H. M. (2016). A distance-based framework to deal with ordinal and additive inconsistencies for fuzzy reciprocal preference relations. Information Sciences, 328, 189-205. https://doi.org/10.1016/j.ins.2015.08.034

$\mathrm{Xu}, \mathrm{Z}$. S. (2005). Deviation measures of linguistic preference relations in group decision making. Omega, 33(3), 249-254. https://doi.org/10.1016/j.omega.2004.04.008

Xu, Z. S. (2013). Group decision making model and approach based on interval preference orderings. Computers \& Industrial Engineering, 64, 797-803. https://doi.org/10.1016/j.cie.2012.12.013

Yu, D. J., \& Xu, Z. S. (2020). Intuitionistic fuzzy two-sided matching model and its application to personnel-position matching problems. Journal of the Operational Research Society, 71(2), 312-321. https://doi.org/10.1080/01605682.2018.1546662

Zadeh, L. A. (1975). The concept of a linguistic variable and its application to approximate reasoning I. Information Sciences, 8, 199-249. https://doi.org/10.1016/0020-0255(75)90036-5

Zhang, Z. M., \& Chen, S. M. (2019). A consistency and consensus-based method for group decision making with hesitant fuzzy linguistic preference relations. Information Sciences, 501, 317-336. https://doi.org/10.1016/j.ins.2019.05.086

Zhang, H. J., Dong, Y. C., Francisco, C., \& Yu, S. (2019). Consensus efficiency in group decision making: A comprehensive comparative study and its optimal design. European Journal of Operational Research, 275(2), 580-598. https://doi.org/10.1016/j.ejor.2018.11.052

Zhang, B. W., Liang, H. M., Gao, Y., \& Zhang, G. Q. (2018a). The optimization-based aggregation and consensus with minimum-cost in group decision making under incomplete linguistic distribution context. Knowledge-Based Systems, 162, 92-102. https://doi.org/10.1016/j.knosys.2018.05.038

Zhang, B. W., Liang, H. M., Zhang, G. Q., \& Xu, Y. F. (2018b). Minimum deviation ordinal consensus reaching in GDM with heterogeneous preference structures. Applied Soft Computing, 67, 658-676. https://doi.org/10.1016/j.asoc.2017.06.016

Zhang, Z. M., \& Pedrycz, W. (2018). Goal programming approaches to managing consistency and consensus for intuitionistic multiplicative preference relations in group decision making. IEEE Transaction on Fuzzy Systems, 26(6), 3261-3275. https://doi.org/10.1109/TFUZZ.2018.2818074

Zhu, B., \& Xu, Z. S. (2018). Probability-hesitant fuzzy sets and the representation of preference relations. Technological and Economic Development of Economy, 24(3), 1029-1040.

https://doi.org/10.3846/20294913.2016.1266529 